LAWRENCE LIVERMORE N A T IO N A L LABORATORY
A Quantitative Comparison of Numerical Methods for the Compressible Euler Equations: Fifth-order WENO and Piecewise-Linear Godunov

J. A. Greenough, W. J. Rider

November 10, 2003

Journal of Computational Physics 
This document was prepared as an account of work sponsored by an agency of the United States Government. Neither the United States Government nor the University of California nor any of their employees, makes any warranty, express or implied, or assumes any legal liability or responsibility for the accuracy, completeness, or usefulness of any information, apparatus, product, or process disclosed, or represents that its use would not infringe privately owned rights. Reference herein to any specific commercial product, process, or service by trade name, trademark, manufacturer, or otherwise, does not necessarily constitute or imply its endorsement, recommendation, or favoring by the United States Government or the University of California. The views and opinions of authors expressed herein do not necessarily state or reflect those of the United States Government or the University of California, and shall not be used for advertising or product endorsement purposes. 


\title{
A Quantitative Comparison of Numerical Methods for the Compressible Euler Equations: Fifth-order WENO and Piecewise-Linear Godunov *
}

\author{
J. A. Greenough \\ Lawrence Livermore National Laboratory \\ Livermore, California 94550 \\ W. J. Rider \\ Los Alamos National Laboratory \\ Los Alamos, New Mexico 87545
}

A numerical study is undertaken comparing a fifth-order version of the weighted essentially non-oscillatory numerical (WENO5) method to a modern piecewise-linear, second-order, version of Godunov's (PLMDE) method for the compressible Euler Equations. A series of one-dimensional test problems are examined beginning with classical linear problems and ending with complex shock interactions. The problems considered are: (1) linear advection of a Gaussian pulse in density, (2) Sod's shock tube problem, (3) the "peak" shock tube problem, (4) a version of the Shu and Osher shock entropy wave interaction and (5) the Woodward and Colella interacting shock wave problem. For each problem and method, run times, density error norms and convergence rates are reported for each method as produced from a common code test-bed.

The linear problem exhibits the advertised convergence rate for both methods as well as the expected large disparity in overall error levels; WENO5 has the smaller errors and an enormous advantage in overall efficiency (in accuracy per unit CPU time). For the nonlinear problems with discontinuities, however, we generally see both first-order self-convergence of error as compared to an exact solution, or when an analytic solution is not available, a converged solution generated on an extremely fine grid. The overall comparison of error levels shows some variation from problem to

\footnotetext{
* This paper is also available as Los Alamos National Laboratory Report LA-UR-02-5640
} 
problem. For Sod's shock tube, PLMDE has nearly half the error, while on the peak problem the errors are nearly the same. For the interacting blast wave problem the two methods again produce a similar level of error with a slight edge for the PLMDE. On the other hand, for the Shu-Osher problem the errors are similar on the coarser grids, but favors WENO by a factor of nearly 1.5 on the finer grids used. In all cases holding mesh resolution constant though, PLMDE is less costly in terms of CPU time by approximately a factor six. If the CPU cost is taken as fixed, that is run times are equal for both numerical methods, then PLMDE uniformly produces lower errors than WENO for the fixed computation cost on the test problems considered here.

\section{INTRODUCTION}

In recent years, new formally high order finite difference methods have been developed that are designed to robustly treat discontinuities by dropping order (upwinding) in the vicinity of a discontinuity while in smooth regions of the flow maintaining high-order formal order of accuracy. Furthermore, these methods have a rigorous mathematical formulation that admits precise numerical analysis in special cases. Among the most recent family of methods are those known as ENO (essentially non-oscillatory) finite difference methods [11]. A generalization and practical improvement of these very successful schemes is the WENO (weighted ENO) method $[13,16]$. In WENO a convex weighted combination of candidate differencing stencils is used to approximate the flux. In this way, the shortcomings of pure ENO methods [21] could be avoided while preserving high order of accuracy (for linear

problems and where the flow is fully resolved), robustness and efficiency. It is worth noting that WENO (and ENO) methods rely on upwinding and, hence, a reduction in order at flow discontinuities in much the same way as the formally second-order, MUSCL methods considered here, which reduce to first-order at flow discontinuities. The specific MUSCL method used for comparison to WENO in this work is a piecewise linear MUSCL method using a direct Eulerian approach (PLMDE) originally devised by Colella [4][28].

PLMDE and the WENO methods attempt to improve upon first order results by introducing some higher-order elements to the schemes. From this perspective, these methods can be termed bottom-up type higher order methods. These methods can be contrasted 
with truly high order methods, such as spectral methods. Spectral methods, when applied to problems with discontinuities, include algorithmic elements that stabilize the solution and mollify the induced Gibbs oscillations. Locally (or globally) there is some reduction from spectral order of accuracy, so these methods are termed top-down type higher-order methods. In the present work these schemes are not considered and we focus attention on the bottom-up methods.

In coupled hyperbolic problems with discontinuities, it was proven by Majda and Osher [17] that numerical methods with modest restrictions are subject to a loss of formal accuracy. The accuracy degenerates to first-order in all, but very special cases between characteristics emanating from a discontinuity. Since that time, other researchers have revisited this issue, but without any evidence to modify Majda and Osher's result. Examples are found by Donat and Osher [9], where sub-cell resolution can overcome this problem. Casper and Carpenter also report similar results [3].

There have been several papers that have included the comparison of different methods. Most notable among these is Woodward and Colella's comparison of methods in 1984 [26]. This includes the introduction to the interacting shock wave problem considered here and the quantitative analysis of results for that problem. Among the methods tested in that paper are an early version of PLMDE and PPMDE, but WENO methods were developed more than a decade after the paper's publication. The comparison includes several multidimensional problems, but detailed quantitative comparison is not possible due to the presence of shear and vortical structures in the flows. Zalesak provided a second set of comparisons for shock capturing methods in 1987 including problems with shocks [27]. Recently, Liska and Wendroff have compared a broad cross-section of modern methods on a variety of shocked flows [15]. In one dimension the comparisons are made quantitatively for problems having analytical solutions. Again, multidimensional problems are not examined quantitatively only qualitatively.

The present work considers a set of one-dimensional test problems of increasing complexity. The idea is to examine the numerical methods in terms of efficiency and accuracy in the linear regime to establish a baseline for performance. As most problems of interest in compressible flow are nonlinear, we consider nonlinear problems that are relatively simple yet still contain interesting structures. The features seen in these one-dimensional problems are prototypical of many features seen in more complex multi-dimensional flows. The analysis 
methods used in the linear case are applied to the nonlinear problems and the results are measured and compared. In order of increasing complexity the test problems are: (1) linear advection of a Gaussian pulse, (2) Sod's shock tube problem [23], (3) the "peak" shock tube problem [15], (4) a version of the Shu and Osher shock entropy wave interaction [22] and (5) the Woodward and Colella interacting shock wave problem [26].

In order to make the comparisons as precise as possible, we calculate normed errors relative to either an exact solution, in the case of linear advection and shock tube problems, or to a highly resolved solution in the other nonlinear tests. When comparison is made to an analytic solution, the errors can be computed and a convergence rate can be derived. This gives the rate at which the computed solution is approaching the true solution. For problems where we do not have an analytic solution, we use two ways to judge the computed solutions. The first is to use the highly resolved solution as the "true" solution and proceed as in the previous case. In this work, we will use an additional numerical method as an independent arbiter for determining the "true" solution. When the resolutions of the computed solutions approach the resolution of the highly resolved one, the errors go to zero and the convergence rates become large. So, for this way of characterizing errors to make sense, the resolutions between the highly resolved "true" solution and the computed ones must be sufficiently different and the errors in the highly resolved solution to be much smaller than those of the coarser computed solution. This gives enough room so that anomalously high convergence rates and low errors are not encountered. Another way to judge computed solutions is to use self-convergence. With this approach, we compare a computed solution to one on a grid twice as fine. This method says nothing about the quality of the solution as compared to the "true" one, but does provide information regarding the internal consistency of the numerical method and its intrinsic convergence properties.

Examining errors and convergence rates is insufficient for a complete comparison of numerical methods. The cost in computational time must also be considered (accuracy per unit CPU time). A common software test-bed has been developed that contains the numerical implementations of the schemes used in this study. This should help assure the reader that neither method has been given an undue advantage due to implementation optimizations. Similarly, we are assured that both methods are implemented in a comparable way and that the code has been compiled in exactly the same way. For the problems considered, we report run times on a single CPU. In real applications, however, single CPU performance is largely 
ignored in favor of parallel efficiency and scaling, but for the purposes of this study, this is the appropriate metric. We note that both methods have similar parallel implementations and data transfer patterns.

Inherently multidimensional problems with shock waves do not readily admit analytical solutions, thus one is left with using grid converged solutions to estimate error. The grid converged solutions used in this paper employ meshes that are at least 8 times finer than the finest grid examined for error. Attempting to meet this sort of criterion in two dimensions would correspond to at least 512 times the computational effort as the finest grid examined for error. In three dimensions the effort grows to 4096. Clearly there is a huge additional amount of effort that must be expended in order to extend one dimensional quantitative comparisons to multiple spatial dimensions.

One dimensional problems provide a look at a particular method's performance in an idealized configuration. That is as all embedded discontinuities are grid-aligned in one dimension, numerical dissipation and contact smearing is minimized. Hence one dimensional test problems show a method when at its best. Increasing the inherent dimensionality of the tests only degrades the schemes performance by increasing the intrinsic dissipation due to non-grid aligned effects. Furthermore, the computational cost in multiple dimensions can be determined by simple dimensional scaling from measured one dimensional performance as most modern multi-dimensional numerical methods are constructed from either onedimensional sweeps or a composition of one-dimensional operations.

For multi-dimensional shock driven flows that contain embedded unstable features (e.g. Richtmyer-Meshkov or Kelvin-Helmholtz instability) there are additional issues not mentioned above that make quantitative analysis and comparisons problematic. There is the real issue of how one measures convergence or even what convergence means for these unstable flows. There is also an additional intimately related issue as to what the appropriate set of equations that should be solved are (Euler versus Navier-Stokes) so that convergence can even be realized.

The paper begins with a brief description of the numerical methods as implemented in the test-bed. We provide mainly an overview of the key features of the methods implemented and refer the reader to appropriate references for the details. Next the methods are compared for each of the test problems over a range of grid resolutions. Finally, we summarize the results and state the conclusions. 


\section{COMPUTATIONAL PLATFORM AND CODE OPTIMIZATION}

All of the test problems were run on a IBM T22 laptop using a $750 \mathrm{MHz}$ PIIIM CPU, 512 MB of RAM. The operating system is RedHat Linux 7.1 and Lahey F95 compiler is used (lf95 -0 -tp). Similar results have been obtained on a Compaq ES45 equipped with four $1 \mathrm{GHz}$ EV6.8 Alpha CPUs and 32GB of main memory running Compaq OSF1 V5.1 operating system and the Compaq 190 compiler (-03) but are not reported in the interest of brevity. Tests using different levels of optimization did not change the numbers reported here appreciably so they should be regarded as representative of the performance of the test bed code.

All of the numerical methods described in this paper are implemented in the same software test bed following a similar programming paradigm. The same input and output routines as well as timing routines are used for both numerical methods. These attributes help to minimize any unintentional performance biases in this work.

\section{NUMERICAL METHODS}

Two numerical methods are considered here. The first is the WENO method described in [13] and the references contained therein. We use the total variation diminishing (TVD) third-order Runge-Kutta time integration method [10, 20] and the fifth-order spatial discretization. In the literature this method is given the designator RK3-WENO5. Since this is the only variant of WENO considered here, we simply refer to it as WENO. The thirdorder Runge-Kutta method, while linearly stable up to a CFL number of 1.43, is TVD or (Strongly Stability Preserving) SSP for much smaller numbers. We will use the commonly employed value of 0.6 for all of our results presented here. The basic building block for the present scheme is based on a characteristic projection using the arithmetic mean of the primitive variables [1] for the positive and negative fluxes used in the flux splitting. This

is equivalent to using the conservative variables [12]. This is only slightly different than the approach given in [13] where they consider a Lax-Friedrichs (LF) or a Roe flux (RF) splitting is considered. We note that the present method should be slightly less diffusive than WENO using LF or RF. Other than the precise form of the characteristic projection, our method is identical to the standard WENO implementation. 
The second method is a piecewise linear, second-order version of Godunov's method (PLMDE). This method is the modern version of the original scheme developed by Colella [4], which follows the original development of the MUSCL scheme of van Leer [25]. Formally, the method is second-order in space and time following a predictor-corrector formulation. Predicted values are obtained by a characteristic tracing of the initial data in space-time. That is, cell-centered data, represented by a linear polynomial approximation over the cell, are traced to cell edges at the half-time level (i.e., $\mathrm{t}+\Delta t / 2$ ). This method is stable up to a CFL number of one but we will take a more conservative limit of 0.9 for all of our results. A monotonicity-preserving central-difference approximation is required for the tracing. This so-called slope (first derivative of the reconstruction) is given by the formulation in [4], where a fourth-order slope is used before limiting rather than simply a second-order one (also see [12]) A projection operator [2, 5, 18, 19] completes the tracings, filtering out characteristics that do not contribute to the state at cell edges. This gives left and right states that are input to the Riemann solver. An approximate Riemann solver [6, 18] is used, which solves the Riemann problem for gas dynamics in one space dimension according to the general design given in [2]. The solution to the Riemann problem gives the predicted values. These are used to construct fluxes, which are required to update the solution to the next time step (i.e., $\mathrm{t}+\Delta t)$ in a fully conservative manner. Several additional techniques that are commonly used such as shock flattening and Lapidus viscosity are not utilized here. Sensitivity studies show that the results are insensitive to these algorithmic details and differences in PLMDE solutions are minor.

The algorithm we use to generate highly resolved solutions for the Shu and Osher test problem and the interacting shock wave problem is the piecewise parabolic MUSCL direct Eulerian (PPMDE) scheme with the enhanced monotonicity constraints as defined by Suresh and Huynh [24]. It is formally third-order in space and second-order in time. We choose to use a third method for generating a highly resolved solution to avoid potentially biasing of the results in favor WENO or PLMDE. These results are found on meshes sufficient to produce errors that are at least an order of magnitude lower than PLMDE or WENO results. 


\section{ERROR ANALYSIS}

For the purposes of analyzing the errors quantitatively, we define two error norms used in this study. The first is the $L_{1}$ error norm defined as

$$
E_{L_{1}}=\frac{1}{N} \sum_{i=1}^{N} E(i)
$$

with

$$
E(i)=\frac{\left|S(i)-S_{c}(i)\right|}{\left|S_{c}(i)\right|}
$$

where $S(i)$ is the computed solution available at grid points $i, S_{c}(i)$ is the "true" solution to the problem. If an analytic solution is available, then $S_{c}(i)$ is that solution evaluated at the same points as $S(i)$. If an analytic solution is not available, then we regard a highly resolved calculation as the true solution. $S_{c}(i)$ is conservatively averaged to the same grid that $S(i)$ exists on. This operation is only conducted on conserved quantities consistent with a weak solution.

The other error norm used is the $L_{\infty}$ norm defined as

$$
E_{L_{\infty}}=\max _{(1, \ldots, N)} E(i)
$$

for $S(i)$ and $S_{c}(i)$ as above. As a general comment on these error norms, the $L_{1}$ gives all differences over the domain the same weight. It tends to give a more global integrated view of the errors in a computed solution. The $L_{\infty}$ norm on the other hand emphasizes errors that occur at peaks or at discontinuities. These are very complementary norms and together provide a reasonably complete picture of the errors and make precise what can be visualized by direct comparison of computed solutions. 


\section{TEST PROBLEMS}

\section{A. Linear Advection}

Linear advection of some form is the ubiquitous test for numerical methods. Advection of different shapes have appeared previously in the literature and we reproduce here results for a particular Gaussian pulse. It also serves as means of verifying that the methods as implemented in the test-bed code produce the expected formal order of accuracy. This is the simplest problem we treat and because of linearity is the best understood from the point-of-view of analysis.

\section{Problem Specification}

Consider the linear advection equation $\rho_{t}+u \rho_{x}=0$ in one-dimension with $u>0$ a constant. Here we take a uniform velocity profile, with $u=1$ and take $\rho$ in the shape of a Gaussian profile given by

$$
\rho=1+\exp \left(\left(-r^{2}\right) /\left(2 \delta^{2}\right)\right)
$$

where $r$ is the distance $|x-0.5|$. Here we set $\delta=0.0625$ and define the profile on the domain $0<x<1$. The boundary conditions at $x=0$ and $x=1$ are periodic. We compute the solution to $t=2$ which is two rotations through the domain. We take special note that this problem is solved in the context of the full Euler equations so that the accuracy of the full solver is tested. Our problem specification is completed by specifying the pressure, $p=10$ and $\gamma=1.4$.

\section{Error Analysis}

In this section we compare the errors between the computed solutions and the analytic solution at the same resolution. Table I shows the error norms and rates of convergence for PLMDE. We see that the second-order rate is achieved for the $L_{1}$ norm and first-order in the $L_{\infty}$ norm. The first-order $L_{\infty}$ norm shows the effect of the slope limiter in PLMDE that reduces the slope approximation used in the characteristic tracing to first order when monotonicity is threatened by local extrema. In the table II the $L_{1}$ and $L_{\infty}$ relative errors are shown for the WENO method. The expected convergence rate, fifth-order, is essentially 
achieved for $C F L=0.6$. We note that only $75 \%$ to $80 \%$ of that rate is achieved for the higher $C F L=1$, as shown in table III, providing a justification for taking care not to choose the higher $C F L$ number.

Recall that the $L_{\infty}$ norm emphasizes errors where they are the largest while $L_{1}$ represents an integrated measure; all errors are given equal weight and summed over the entire domain. If we plot the difference, $E(i)=\left|S(i)-S_{c}(i)\right| / S_{c}(i)$, used to construct the norms as a function of grid point, then we can visualize where the errors are largest as well as their spatial structure. In figure 1, we see that for PLMDE the largest errors are near the peak $(x=0.5)$ of the Gaussian, and fall off in a way consistent with the WENO errors. At the peak the errors for both methods are the largest, but those for WENO are nearly two decades smaller in magnitude at the same resolution. For finer meshes the differences become even more extreme owing to the fact that WENO is converging at its designed fifth-order accuracy.

TABLE I: $L_{1}$ and $L_{\infty}$ errors and convergence rates for PLMDE for the advection of a Gaussian pulse at different grid resolutions.

\begin{tabular}{|c|c|c|c|c|}
\hline $\mathrm{N}$ & $E_{L_{1}}$ & $L_{1}$ Rate & $E_{L_{\infty}}$ & $L_{\infty}$ Rate \\
\hline 100 & $1.97 \mathrm{e}-03$ & - & $1.17 \mathrm{e}-02$ & - \\
\hline 200 & $5.64 \mathrm{e}-04$ & 1.80 & $5.19 \mathrm{e}-03$ & 1.17 \\
\hline 400 & $1.50 \mathrm{e}-04$ & 1.91 & $2.33 \mathrm{e}-03$ & 1.16 \\
\hline
\end{tabular}

TABLE II: $L_{1}$ and $L_{\infty}$ errors and convergence rates for the WENO method for the advection of a Gaussian pulse at different grid resolutions using $C F L=0.6$.

\begin{tabular}{|c|c|c|c|c|}
\hline $\mathrm{N}$ & $E_{L_{1}}$ & $L_{1}$ Rate & $E_{L_{\infty}}$ & $L_{\infty}$ Rate \\
\hline 100 & $3.29 \mathrm{e}-05$ & - & $1.27 \mathrm{e}-04$ & - \\
\hline 200 & $1.09 \mathrm{e}-06$ & 4.92 & $5.31 \mathrm{e}-06$ & 4.58 \\
\hline 400 & $3.69 \mathrm{e}-08$ & 4.88 & $2.11 \mathrm{e}-07$ & 4.65 \\
\hline
\end{tabular}


TABLE III: $L_{1}$ and $L_{\infty}$ errors and convergence rates for the WENO method for the advection of a Gaussian pulse at different grid resolutions using $C F L=1.0$.

\begin{tabular}{|c|c|c|c|c|}
\hline $\mathrm{N}$ & $E_{L_{1}}$ & $L_{1}$ Rate & $E_{L_{\infty}}$ & $L_{\infty}$ Rate \\
\hline 200 & $5.662 \mathrm{e}-06$ & - & $3.247 \mathrm{e}-05$ & - \\
\hline 400 & $2.343 \mathrm{e}-07$ & 4.6 & $1.583 \mathrm{e}-06$ & 4.36 \\
\hline 800 & $1.744 \mathrm{e}-08$ & 3.75 & $1.062 \mathrm{e}-07$ & 3.9 \\
\hline
\end{tabular}

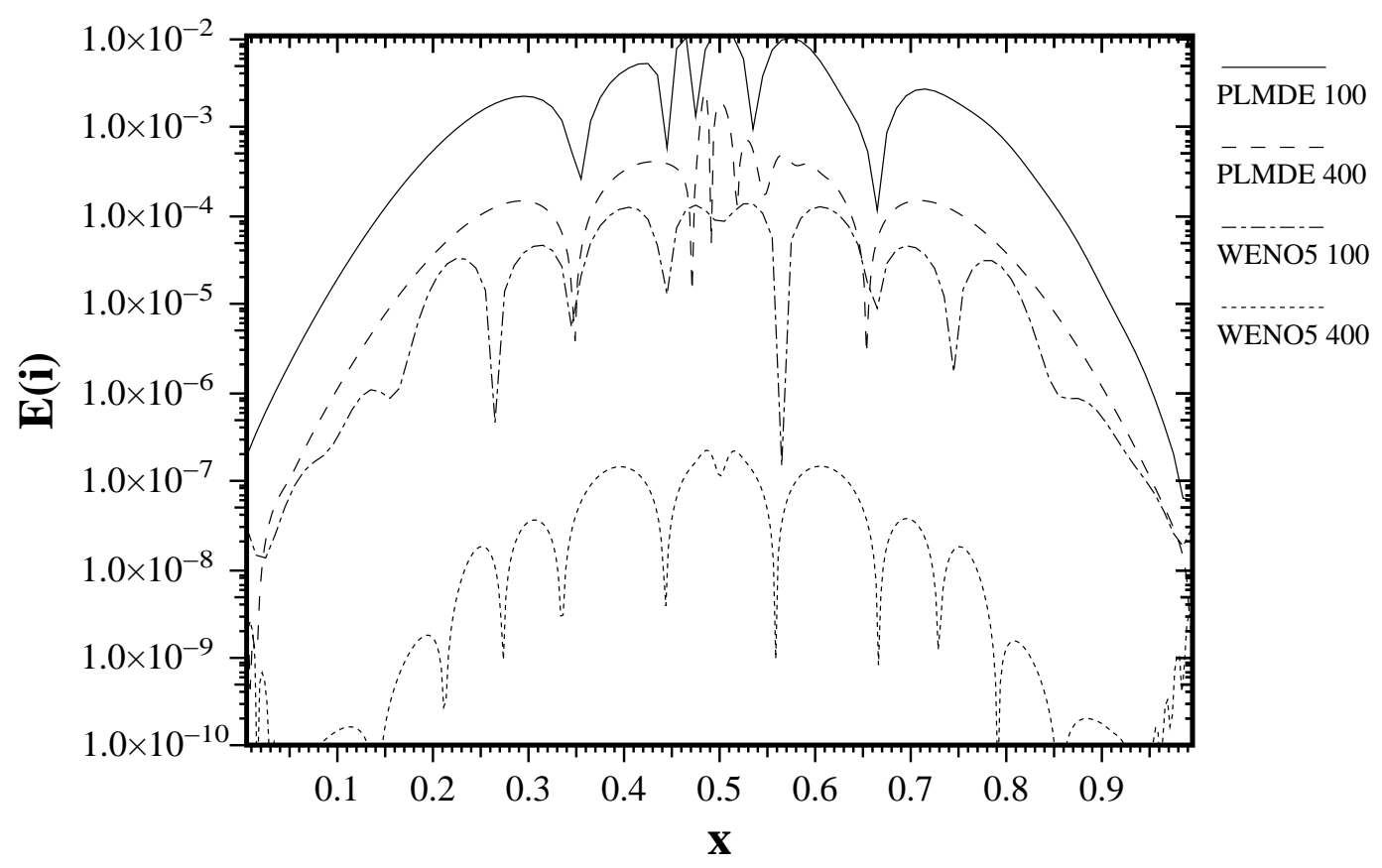

FIG. 1: The quantity $E(i)$ is plotted for PLMDE and the WENO method on a vertical log scale at resolutions indicated in the figure legend.

\section{Base Timings}

In Table IV we show the run time results for the two methods for two periods through the grid. According to the above results, WENO's convergence rate is sensitive to $C F L$. Furthermore, results in the literature uniformly use a $C F L$ value less than 0.6, even though the third-order Runge-Kutta time integration method is stable to a $C F L$ value of 1.43 . In the interests of balancing high accuracy (low errors) and consistency with the existing body of work, we will use the $C F L=0.6$. Note that the timings can be scaled to a different CFL 
using the number of total time steps taken but taking care to note that the relative accuracy of the resultant solution will possibly be degraded.

TABLE IV: Timings for PLMDE and WENO methods for linear advection of a Gaussian pulse. The Ratios column shows the WENO result divided by the PLMDE result.

\begin{tabular}{|c|c|c|c|c|c|}
\hline $\mathrm{N}$ & PLMDE (sec) & time steps & WENO (sec) & time steps & Ratio \\
\hline 100 & 2.11 & 1083 & 11.13 & 1610 & 5.22 \\
\hline 200 & 7.03 & 2137 & 43.40 & 3191 & 6.17 \\
\hline 400 & 24.69 & 4245 & 164.76 & 6352 & 6.67 \\
\hline
\end{tabular}

\section{B. Sod and Peak Shock Tubes}

An equally ubiquitous test for these numerical methods are shock tubes. We will examine two different shock tube problems here, Sod's [23] and the "peak" problem used by Liska and Wendroff [14]. Sod's shock tube is a relatively easy problem, but the peak is quite difficult because of the large density and small distance between the shock and the contact. Both of these problems have analytic solutions, as do all shocktube problems for polytropic gases, providing unambiguous error analysis.

\section{Problem Specifications}

Both problems are specified using a shock tube initial condition: a discontinuous initial state that forms a self-similar profile in the $x / t$ coordinate. For both problems we will provide the left and right initial states in the form of a vector, $(\rho, u, p)^{T}$. In both cases the pressure is related to the density and energy through an ideal gas equation of state with $\gamma=1.4$. In the case of Sod's shock tube the left state $(x<0.5)$ is $(1,0,1)^{T}$ and the right state $(x>0.5)$ is $(0.125,0,0.1)^{T}$. The domain is $x \in[0,1]$ and results are reported at $t=0.2$. We will report results for grids of 100, 200 and 400 cells. The peak problem is defined in the same manner with the left state $\left(x<0.5\right.$ being $(0.1261192,8.9047029,782.92899)^{T}$ and the right state $(x>0.5)$ is $(6.591492,2.2654207,3.1544874)^{T}$ on the unit interval. The problem 
is run to $t=0.0039$. We will report results for grids of 200, 400 and 800 cells. In all cases we ramp up the time step size from one-tenth the CFL limit, specified previously for each method, at a rate of 1.05 per time step until the full specified CFL limit is reached.

\section{Error Analysis}

In this section we compare the errors between the computed solutions and the analytic solution at the same resolution. For Sod's shock tube the $L_{1}$ norm errors for PLMDE are roughly half the size of those for WENO as seen by comparing the errors in tables VI and V. The $L_{\infty}$ norms are comparable in size with the PLMDE errors being slightly smaller. This norm also grows in magnitude in response to the localization of error at the shock.

A plot of the error as a function of $x$ is shown in figure 2. The spatial distribution of the errors for the two methods are very similar; however, in the rarefaction region, $0.25 \leq x \leq$ 0.45, PLMDE has uniformly lower errors. At the shock, $x \approx 0.87$, the WENO errors extend past the shock location while the PLMDE errors drop off sharply. This effect could be the result of the (relative) phase error in the WENO method.

TABLE V: $E_{L_{1}}$ and $E_{L_{\infty}}$ errors and convergence rates for PLMDE on Sod's shock tube at different grid resolutions.

\begin{tabular}{|c|c|c|c|c|}
\hline $\mathrm{N}$ & $E_{L_{1}}$ & $L_{1}$ Rate & $E_{L_{\infty}}$ & $L_{\infty}$ Rate \\
\hline 100 & $8.22 \mathrm{e}-03$ & - & $0.22 \mathrm{e}-00$ & - \\
\hline 200 & $4.48 \mathrm{e}-03$ & 0.88 & $0.25 \mathrm{e}-00$ & -0.20 \\
\hline 400 & $2.62 \mathrm{e}-03$ & 0.77 & $0.33 \mathrm{e}-00$ & -0.37 \\
\hline
\end{tabular}

The peak shock tube places a high premium on computing a narrow density peak. The errors for these methods on this problem is quite similar, as is evident from the data shown in Tables VII and VIII. The convergence rates are poor and irregular, but similar. At the 
TABLE VI: $E_{L_{1}}$ and $E_{L_{\infty}}$ errors and convergence rates for the WENO method for Sod's shock tube at different grid resolutions.

\begin{tabular}{|c|c|c|c|c|}
\hline $\mathrm{N}$ & $E_{L_{1}}$ & $L_{1}$ Rate & $E_{L_{\infty}}$ & $L_{\infty}$ Rate \\
\hline 100 & $1.58 \mathrm{e}-02$ & - & $0.37 \mathrm{e}-00$ & - \\
\hline 200 & $8.24 \mathrm{e}-03$ & 0.93 & $0.40 \mathrm{e}-00$ & -0.01 \\
\hline 400 & $4.47 \mathrm{e}-03$ & 0.88 & $0.46 \mathrm{e}-00$ & -0.18 \\
\hline
\end{tabular}

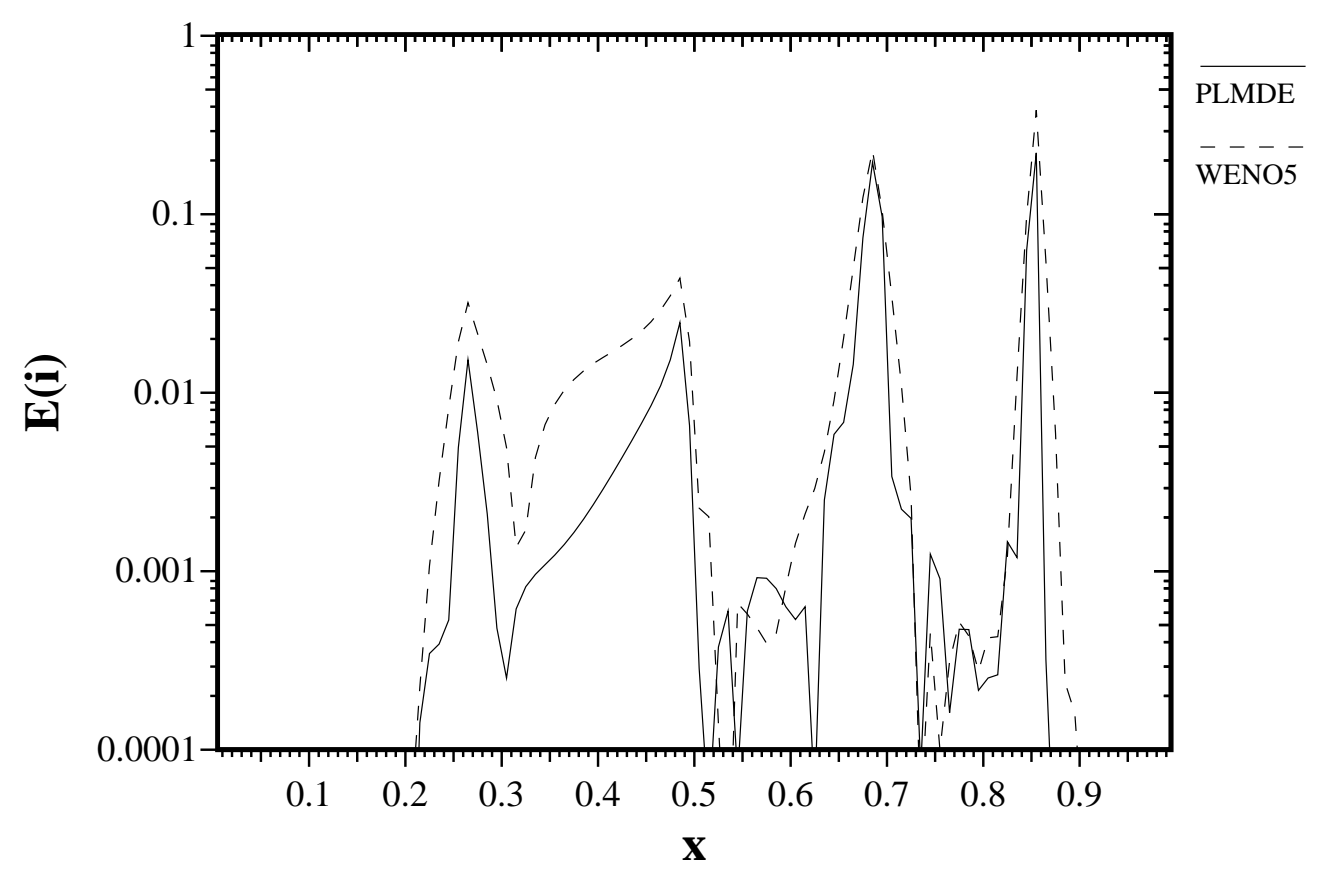

FIG. 2: The quantity $E(i)$ for density on Sod's shock tube plotted for PLMDE (solid line) and the WENO method (dashed line) on a vertical log scale for $N=100$.

finest resolution used the solutions in the vicinity of the peak density are nearly identical as shown in Figure 3. The differences favor PLMDE with its solution being slightly sharper although slightly lower in maximum density at the finest (800 cell) resolution. The point of this problem is to demonstrate the similarity of performance of the two schemes on this difficult problem. 
TABLE VII: $L_{1}$ and $L_{\infty}$ errors and convergence rates for PLMDE for the peak shock tube at different grid resolutions.

\begin{tabular}{|c|c|c|c|c|}
\hline $\mathrm{N}$ & $E_{L_{1}}$ & $L_{1}$ Rate & $E_{L_{\infty}}$ & $L_{\infty}$ Rate \\
\hline 100 & 1.02 & - & 139. & - \\
\hline 200 & 0.86 & 0.24 & 187. & -0.43 \\
\hline 400 & 0.29 & 1.57 & 120. & 0.65 \\
\hline
\end{tabular}

TABLE VIII: $L_{1}$ and $L_{\infty}$ errors and convergence rates for the WENO method for the peak shock tube at different grid resolutions.

\begin{tabular}{|c|c|c|c|c|}
\hline $\mathrm{N}$ & $E_{L_{1}}$ & $L_{1}$ Rate & $E_{L_{\infty}}$ & $L_{\infty}$ Rate \\
\hline 100 & 0.93 & - & 125. & - \\
\hline 200 & 0.86 & 0.12 & 185. & -0.57 \\
\hline 400 & 0.29 & 1.55 & 129. & 0.52 \\
\hline
\end{tabular}

\section{Base Timings}

In Tables IX and X we show the run time results for the two methods. The CFL limits are as before. The relative cost of the two methods for this simple nonlinear problem is the essentially the same as that for the linear advection problem. That is, the relative cost of WENO to PLMDE is approximately six.

\section{Shu and Osher Test Problem}

This test problem is probably the simplest one-dimensional compressible flow problem that contains interesting structures. It is also considered to be a good one-dimensional surrogate for the interaction of a shock wave with a turbulent field. 


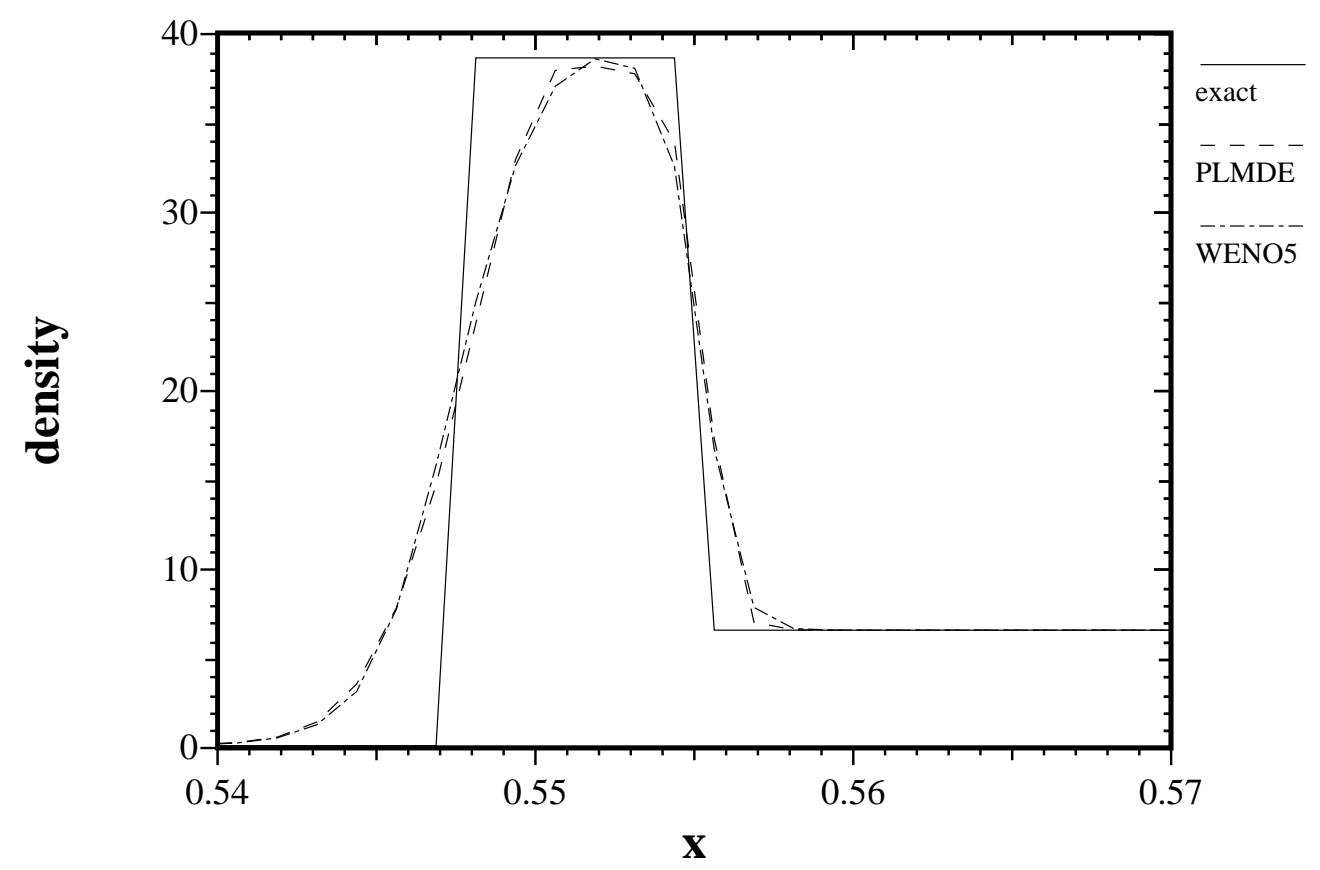

FIG. 3: The density around the peak in the peak shock tube problem is plotted for PLMDE (dashed line) and the WENO method (dot-dashed line) with the exact solution (solid line) for $N=800$.

TABLE IX: Timings for PLMDE and WENO methods for Sod's shock tube. The Ratios column shows the WENO result divided by the PLMDE result.

\begin{tabular}{|c|c|c|c|c|c|}
\hline $\mathrm{N}$ & PLMDE (sec) & time steps & WENO (sec) & time steps & Ratios \\
\hline 100 & 0.17 & 72 & 0.69 & 95 & 4.06 \\
\hline 200 & 0.43 & 127 & 2.34 & 173 & 5.44 \\
\hline 400 & 1.40 & 238 & 8.98 & 329 & 6.41 \\
\hline
\end{tabular}

TABLE X: Timings for PLMDE and WENO methods for the peak shock tube. The Ratios column shows the WENO result divided by the PLMDE result.

\begin{tabular}{|c|c|c|c|c|c|}
\hline $\mathrm{N}$ & PLMDE (sec) & time steps & WENO (sec) & time steps & Ratios \\
\hline 200 & 2.37 & 168 & 0.43 & 121 & 5.51 \\
\hline 400 & 8.57 & 305 & 1.40 & 212 & 6.12 \\
\hline 800 & 35.78 & 579 & 5.00 & 395 & 7.15 \\
\hline
\end{tabular}




\section{Problem Definition}

The problem is a Mach 3 shock wave in a $\gamma=1.4$ perfect gas interacting with upstream sinusoidal density waves. Specifically at $t=0$,

$$
\begin{gathered}
x<-4: \rho=3.857143 ; \rho u=2.629369 ; p=10.33333 \\
x \geq-4: \rho=1-\epsilon \sin (\lambda x) ; \rho u=0 . ; p=1
\end{gathered}
$$

defined on $-5<x<5$. In the present study we take $\epsilon=0.2$ and $\lambda=5 \pi$. For a different set of values see [13]. We choose this set because they give very high frequency oscillations in the near post-shock region that transitions downstream to a longer wavelength "N-wave" pattern. We wish to stress the algorithms in their abilities to accurately resolve high frequency modes after shock processing. The boundary conditions are set as inflow/outflow at $x=-5$ and $x=5$. The final time for analysis is taken to be $t=1.8$, at which time, there is no flow across the $x=5$ boundary.

For the "exact" solution we use an extensively refined grid solution using a different method. We choose PPMDE embellished with relaxed monotonicity requirements as defined by Suresh and Huynh [24] to produce this solution using 25600 cells. This method combines elements of both the PLMDE and WENO5 methods without being directly derived from either. This is to avoid making comparisons that are overly biased for or against either method. Furthermore, we will now provide evidence that the errors in the highly converged solution are sufficiently well resolved to be considered as a standard. In Table XI we display the $L_{1}$ and $L_{\infty}$ errors for this solution as well as the self-convergence rates. The $L_{1}$ errors are a factor of approximately 10 times lower than those estimated for PLMDE and WENO5 below. In the case of the $L_{\infty}$ errors this factor is also approximately 10 .

At this point it is worthwhile to discuss two manners of estimating error and determining convergence rates. One method uses "self-convergence" where a method's next most refined grid is used to estimate errors rather than an exact or surrogate of an exact solution. Our principle tool in this paper is an exact solution as determined by an extremely refined grid. In this section we will also report self-convergence, which, in a sense, marks a method's internal progress toward a solution rather than the absolute measure of such progress. 
TABLE XI: $L_{1}$ and $L_{\infty}$ Errors for the highly converged PPMDE solution of the Shu-Osher test problem.

\begin{tabular}{|c|c|c|c|c|}
\hline $\mathrm{N}$ & $E_{L_{1}}$ & $L_{1}$ Rate & $E_{L_{\infty}}$ & $L_{\infty}$ Rate \\
\hline $6400: 12800$ & $3.00 \mathrm{e}-04$ & - & $9.65 \mathrm{e}-02$ & - \\
\hline $12800: 25600$ & $1.24 \mathrm{e}-04$ & 1.27 & $2.84 \mathrm{e}-02$ & 1.76 \\
\hline
\end{tabular}

\section{Error Analysis}

Examining the errors quantitatively shows the quality of the computed solution for a given resolution. In Table XII is shown the $L_{1}$ errors for PLMDE and WENO for the grid resolutions considered. We use the solution at $N=25600$ generated using PPMDE as the true solution for the error analysis on this test problem. We see in Table XII that the errors between the two methods are comparable at similar resolution for the coarser resolutions, $N<1600$. Also for these cases, the convergence rate is essentially zeroth order. The poor convergence rates are a bit puzzling at first glance in light of the convergence rates published in [26] for the next test problem. They show first-order convergence for nominally secondorder codes and less than first-order convergence for the first-order version of Godunov's method. An explanation for this could be the nature of this test problem. There is a minimum resolution required to represent the high frequency modes present in the postshock region. Less than expected convergence rates would indicate that the low resolution computed solutions are very different (uncorrelated) from the highly resolved solution.

For $N \geq 1600$, the magnitude of the errors differs by less than a factor of two in favor of WENO with both schemes showing better than first order convergence. We are now in the regime where the computed solutions are correlated with the highly resolved solution.

The $L_{1}$ self-convergence errors are summarized in table XIII. Below the critical resolution of $N=1600$ we see nominally first order convergence between the two methods. As the resolution matches the critical resolution in the $N=800: 1600$ case, we observe divergence. For the $N=1600: 3200$ case the solutions appear to converge again at nominally first order or better. The convergence rates when considering self-convergence indicate the rate at which a computed solution is approaching a solution on a grid twice as fine. It makes no statement regarding the accuracy of the computed solution relative to the true solution.

The $L_{\infty}$ errors are shown in Tables XIV and XV. For both methods, the errors are 
essentially oscillatory throughout the resolutions considered and of similar magnitudes for both methods. When examining the $L_{\infty}$ errors under self-convergence, we observe a similar behavior with respect to PLMDE. For WENO, the errors are roughly constant for $N \leq 1600$. At higher resolutions, the errors then decrease.

TABLE XII: $L_{1}$ errors for PLMDE and WENO relative to the $\mathrm{N}=25,600$ PPMDE solution for the Shu and Osher test problem.

\begin{tabular}{|c|c|c|c|c|}
\hline $\mathrm{N}$ & $E_{L_{1}}$ PLMDE & PLMDE $L_{1}$ Rate & $E_{L_{1}}$ WENO & WENO $L_{1}$ Rate \\
\hline 200 & $1.858 \mathrm{e}-02$ & - & $2.104 \mathrm{e}-02$ & - \\
\hline 400 & $1.837 \mathrm{e}-02$ & 0.16 & $1.959 \mathrm{e}-02$ & 0.10 \\
\hline 800 & $1.669 \mathrm{e}-02$ & 0.14 & $1.626 \mathrm{e}-02$ & 0.27 \\
\hline 1600 & $7.018 \mathrm{e}-03$ & 1.25 & $4.001 \mathrm{e}-03$ & 2.02 \\
\hline 3200 & $2.423 \mathrm{e}-03$ & 1.53 & $1.358 \mathrm{e}-03$ & 1.60 \\
\hline
\end{tabular}

TABLE XIII: $L_{1}$ errors for PLMDE and WENO using self-convergence for the Shu and Osher test problem.

\begin{tabular}{|c|c|c|c|c|}
\hline $\mathrm{N}$ & $E_{L_{1}}$ PLMDE & PLMDE $L_{1}$ Rate & $E_{L_{1}}$ WENO & WENO $L_{1}$ Rate \\
\hline $200: 400$ & $7.198 \mathrm{e}-03$ & - & $6.137 \mathrm{e}-03$ & - \\
\hline $400: 800$ & $3.348 \mathrm{e}-03$ & 1.10 & $4.401 \mathrm{e}-03$ & 0.48 \\
\hline $800: 1600$ & $1.077 \mathrm{e}-02$ & -0.14 & $1.275 \mathrm{e}-02$ & -1.53 \\
\hline $1600: 3200$ & $6.125 \mathrm{e}-03$ & 0.81 & $2.826-03$ & 2.17 \\
\hline
\end{tabular}

\section{Base Timings}

Table XVI summarizes the run times in seconds to compute the solution to $t=1.8$ for the two methods on successively finer grids. Our standard CFL is used for both methods. 
TABLE XIV: $L_{\infty}$ errors for PLMDE and WENO relative to the $N=25600$ PPMDE solution for the Shu and Osher test problem.

\begin{tabular}{|c|c|c|}
\hline $\mathrm{N}$ & $E_{L_{\infty}}$ PLMDE & $E_{L_{\infty}}$ WENO \\
\hline 200 & 0.429 & 0.902 \\
\hline 400 & 0.188 & 0.667 \\
\hline 800 & 0.290 & 0.369 \\
\hline 1600 & 0.185 & 0.572 \\
\hline 3200 & 0.230 & 0.399 \\
\hline
\end{tabular}

TABLE XV: $L_{\infty}$ errors for PLMDE and WENO using self-convergence for the Shu and Osher test problem.

\begin{tabular}{|c|c|c|}
\hline $\mathrm{N}$ & $E_{L_{\infty}}$ PLMDE & $E_{L_{\infty}}$ WENO \\
\hline $200: 400$ & 0.143 & 0.274 \\
\hline $400: 800$ & 0.081 & 0.216 \\
\hline $800: 1600$ & 0.238 & 0.216 \\
\hline $1600: 3200$ & 0.077 & 0.100 \\
\hline
\end{tabular}

The ratio column gives the ratio of WENO runtime to that of PLMDE. We see that at a given resolution PLMDE is faster by approximately a factor of six.

TABLE XVI: Run times for PLMDE and WENO for the Shu and Osher test problem.

\begin{tabular}{|c|c|c|c|c|c|}
\hline $\mathrm{N}$ & PLMDE Time (sec) & time steps & WENO Time (sec) & time steps & Ratio \\
\hline 200 & 0.84 & 250 & 4.84 & 334 & 5.76 \\
\hline 400 & 2.89 & 468 & 17.38 & 634 & 6.01 \\
\hline 800 & 11.02 & 903 & 73.07 & 1235 & 6.63 \\
\hline 1600 & 46.09 & 1774 & 280.62 & 2443 & 6.09 \\
\hline 3200 & 226.74 & 3514 & 1278.31 & 4857 & 5.63 \\
\hline
\end{tabular}




\section{Density Field Visualizations}

In this section we examine plots of the computed density fields for WENO and PLMDE. We will divide the flow into four distinct regions for presentation of results: near the shock $x \in[2,2.5]$; the near field (NF), entropy wave (EW) $x \in[1,2]$; the transition to the N-waves $x \in[0,1]$; and the far field $(\mathrm{FF}), \mathrm{N}$-wave region $x \in[-2,0]$. We display the extremely fine grid solution (taken as being nearly "exact") in Figure 4. We then show a series of plots displaying the grid convergence behavior of both schemes and a comparison of errors on the medium size grid (1600 cells). Figures 5 through 16 show these comparisons. In the near shock region we see that the WENO method commits greater errors at the shock, but the errors are smaller once the fluid is processed by the shock. This trend continues in the NF region where the advantage of WENO is the greatest. As the flow transitions to a series of $\mathrm{N}$-waves the errors become nearly equal in magnitude. Finally, the errors in WENO become larger than those committed by PLMDE in the FF region in keeping with our general observation from this data that WENO produces larger errors near a shock wave as compared to PLMDE.

The errors are shown in figure 5 for the NF region. We see that WENO has larger errors in the neighborhood of the shock wave. Behind the shock in the beginning of the entropy wave oscillations, we see that WENO and PLMDE have similar errors with a slight advantage to WENO. The corresponding density plots for $N=800,1600$ and 3200 with the exact solution for reference for PLMDE and WENO are shown in figures 9 and 10, respectively.

In the EW region of the flow, the errors plotted in figure 6 are uniformly lower for WENO compared to PLMDE. Typical differences are roughly a factor of two in this region and these errors dominate the overall error for the problem. If we compare the distribution of errors with the density plot for PLMDE, figure 11, and for WENO, 12, we see that the minimum errors typically occur at local minima in the density while the maximum errors typically occur at local maxima. We also see the dramatic effect that occurs for both methods when the resolution is increased above $N=800$; the wavelength of the high frequency oscillations are beginning to be resolved.

At the end of the EW region, the flow undergoes a transition to N-Waves (TN). The errors in this region for PLMDE and WENO are plotted in figure 7. Similar errors are observed throughout this region for both methods. Figures 13 and 14 show the density field 


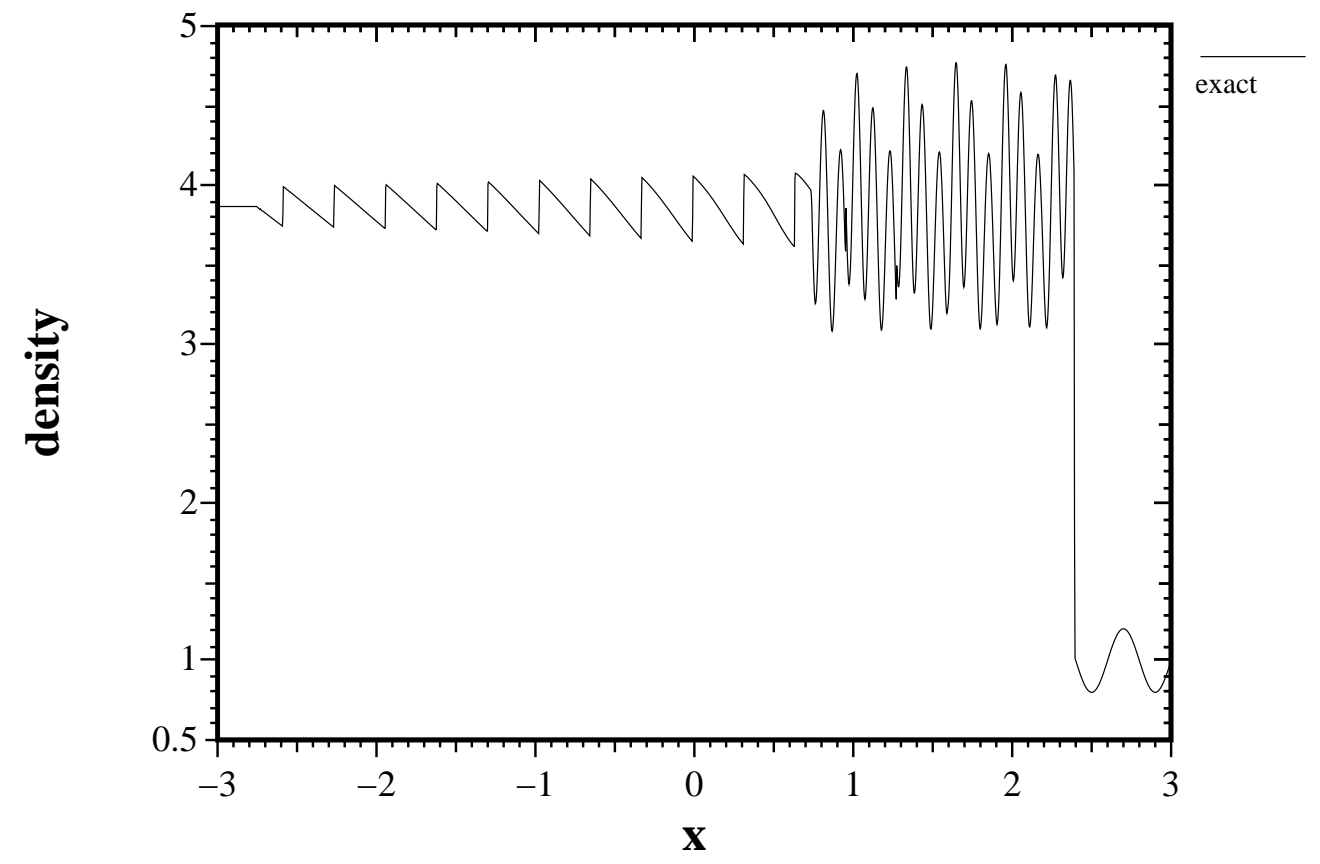

FIG. 4: The "exact" solution to the Shu-Osher problem from PPMDE using 25600 cells. The domain is divided into four distinct regions for presentation of the results: near the shock or nearfield (NF) $x \in[2,2.5]$, the entropy wave (EW) $x \in[1,2]$, the transition to the N-waves (TN) $x \in[0,1]$, and the far-field $(\mathrm{FF}), \mathrm{N}$-wave region $x \in[-2,0]$.

for PLMDE and WENO, respectively, for $N=800,1600$ and 3200 resolutions. The density fields produced by both methods are also similar in this region which is consistent with the similarity of the error plots.

Finally in the FF region, the errors for PLMDE and WENO are shown in figure 8. At the discontinuities of the $\mathrm{N}$-waves, both methods produce similar errors. In the regions in between, PLMDE has an advantage in error magnitude. The errors can be compared with the density fields which are shown in figures 15 and 16 for PLMDE and WENO, respectively. A slight advantage is observed near the peaks for PLMDE that is consistent with the error plot in this region. 


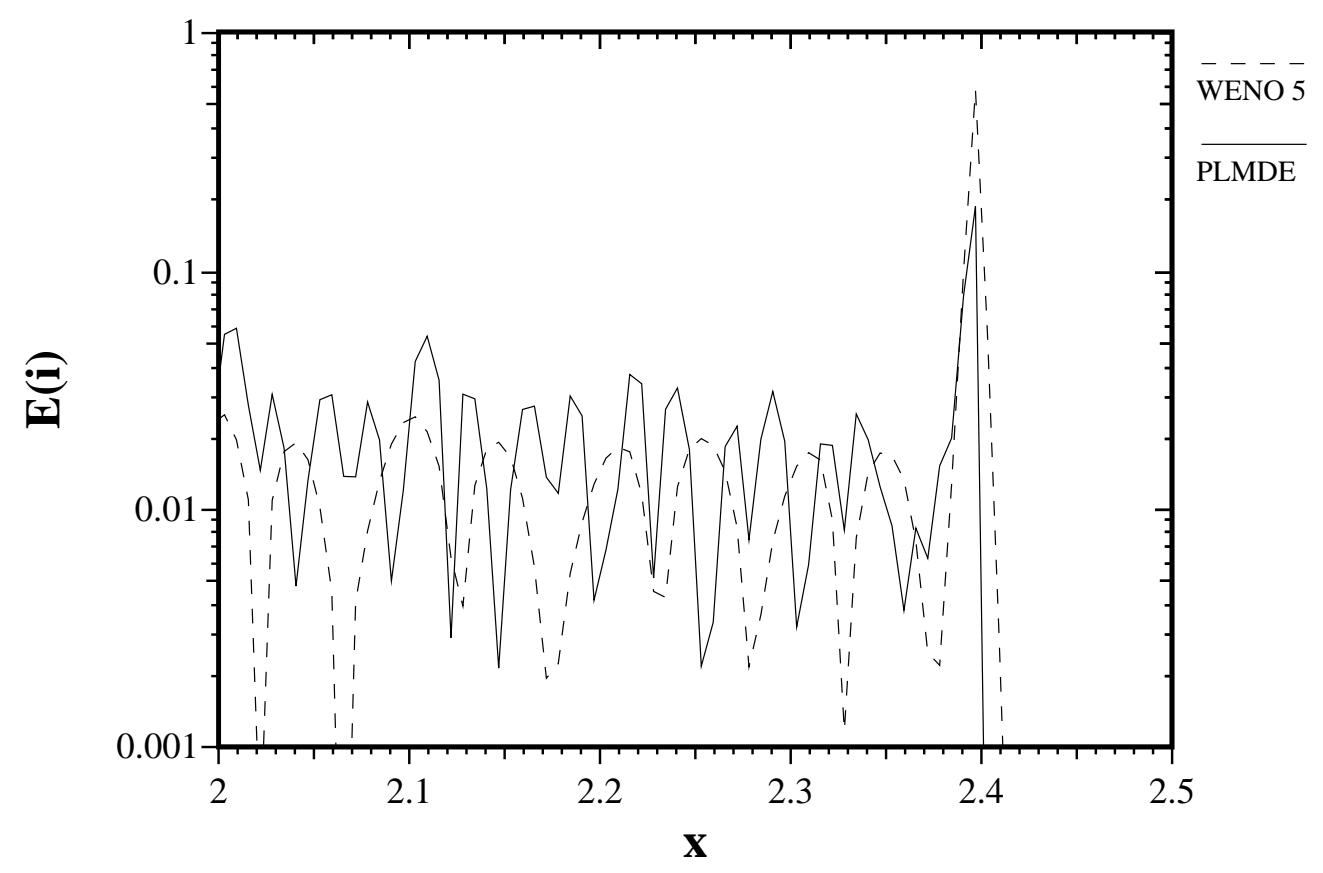

FIG. 5: A comparison of the errors in the near shock (NF) region for the Shu and Osher test problem. PLMDE is shown as a solid line and WENO is shown with a dashed line.

\section{Break-even Analysis}

It is an essential exercise to take the time used by WENO as a fixed resource that we want to fully utilize for a PLMDE calculation. Table XVII shows the PLMDE resolution necessary to approximately equate run times. Overall, we can use a grid more than twice as fine for PLMDE and have the run times equal. This uniformly lowers the errors for each equal-WENO-time PLMDE calculation by approximately a factor of 2.5, assuming first order convergence. Looking back to tables XII and XIV for the $L_{1}$ and $L_{\infty}$ errors, respectively, we see that a doubling of the number of grid points produces a PLMDE solution with lower errors by at least a factor of two for the norms considered here. For this test problem and a fixed amount of computational time, PLMDE can produce a quantitatively more accurate solution independent of the chosen error norm. 


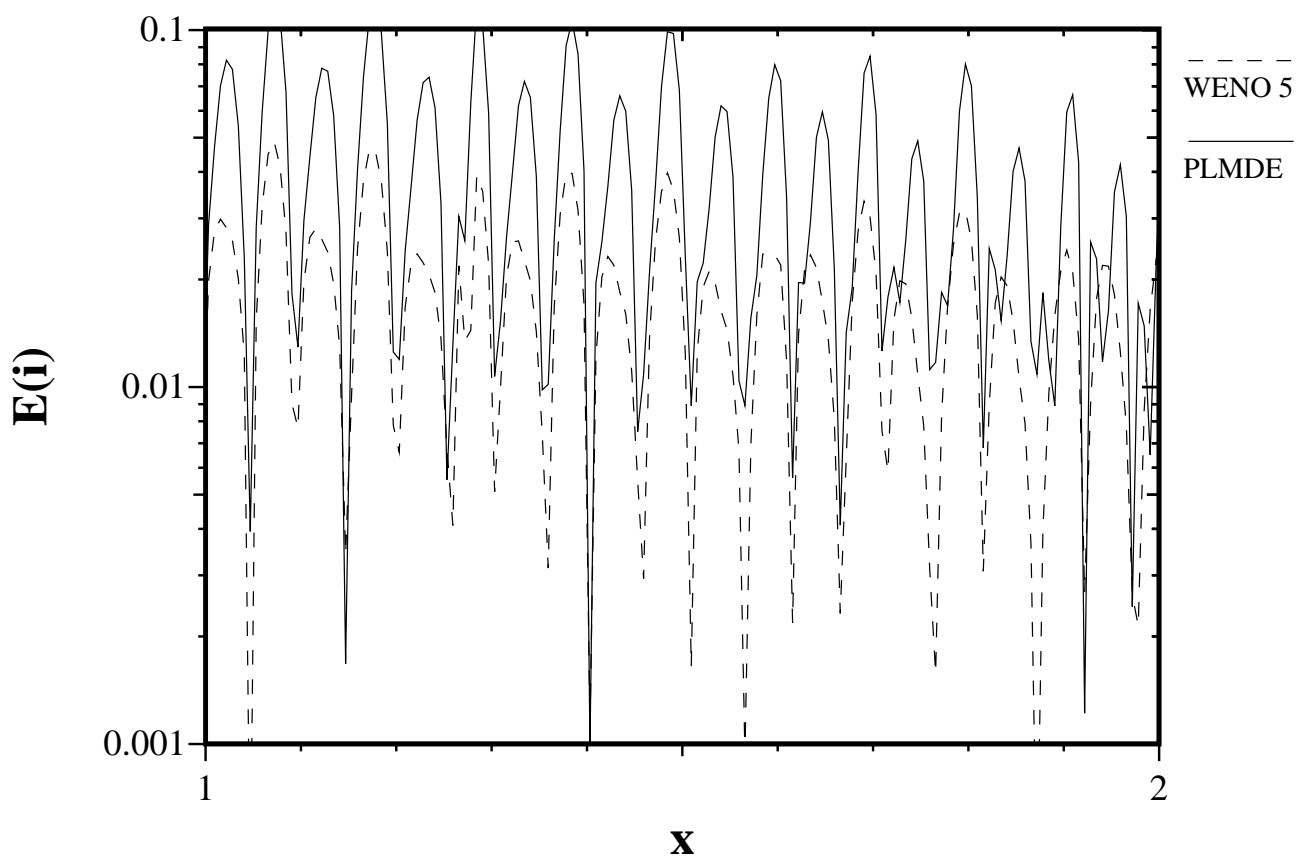

FIG. 6: A comparison of the errors in the entropy wave (EW) region for the Shu and Osher test problem. PLMDE errors are shown as a solid line and WENO errors are shown with a dashed line.

TABLE XVII: Comparison of WENO to PLMDE resolution for equal run times for the Shu and Osher test problem.

\begin{tabular}{|c|c|c|c|}
\hline WENO N & PLMDE N & PLMDE Time (sec) & Ratio \\
\hline 200 & 500 & 4.72 & 0.98 \\
\hline 400 & 1020 & 17.68 & 1.02 \\
\hline 800 & 2020 & 74.14 & 1.01 \\
\hline 1600 & 3940 & 289.10 & 1.03 \\
\hline
\end{tabular}

\section{Woodward-Colella Interacting Shock Wave Problem}

The interacting shock wave problem was first featured as part of test suite in [26]. It is a very challenging problem for Eulerian methods as the compressions are exceptionally high in very small volumes. This repeats some of the features of the "peak" shock tube. Also, it features strong nonlinear effects through the interaction of shock, rarefactions and contact 


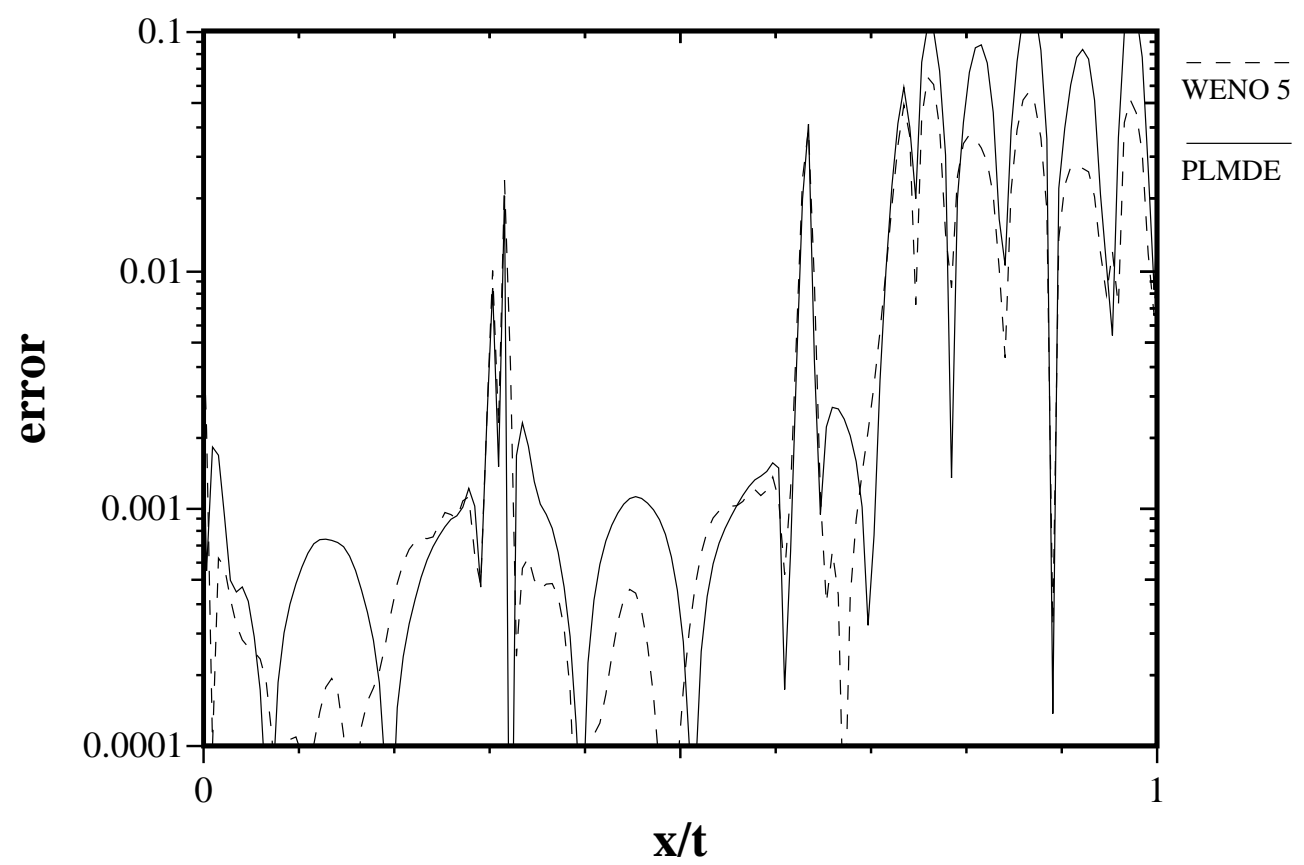

FIG. 7: A comparison of the errors in the transition to N-waves (TN) region for the Shu and Osher test problem. PLMDE errors are shown as a solid line and WENO errors are shown with a dashed line.

discontinuities.

\section{Problem Description}

The one-dimensional interacting shock wave problem is two separate shock tube problems featuring a region of low pressure in between. The fluid is a $\gamma=1.4$ perfect gas. At $t=0$, we have the following configuration:

$$
\begin{gathered}
0<x<0.1: \rho=1 ; \rho u=0 ; p=1000 \\
0.1<x<0.9: \rho=1 ; \rho u=0 . ; p=0.01 \\
0.9<x<1: \rho=1 ; \rho u=0 . ; p=100
\end{gathered}
$$




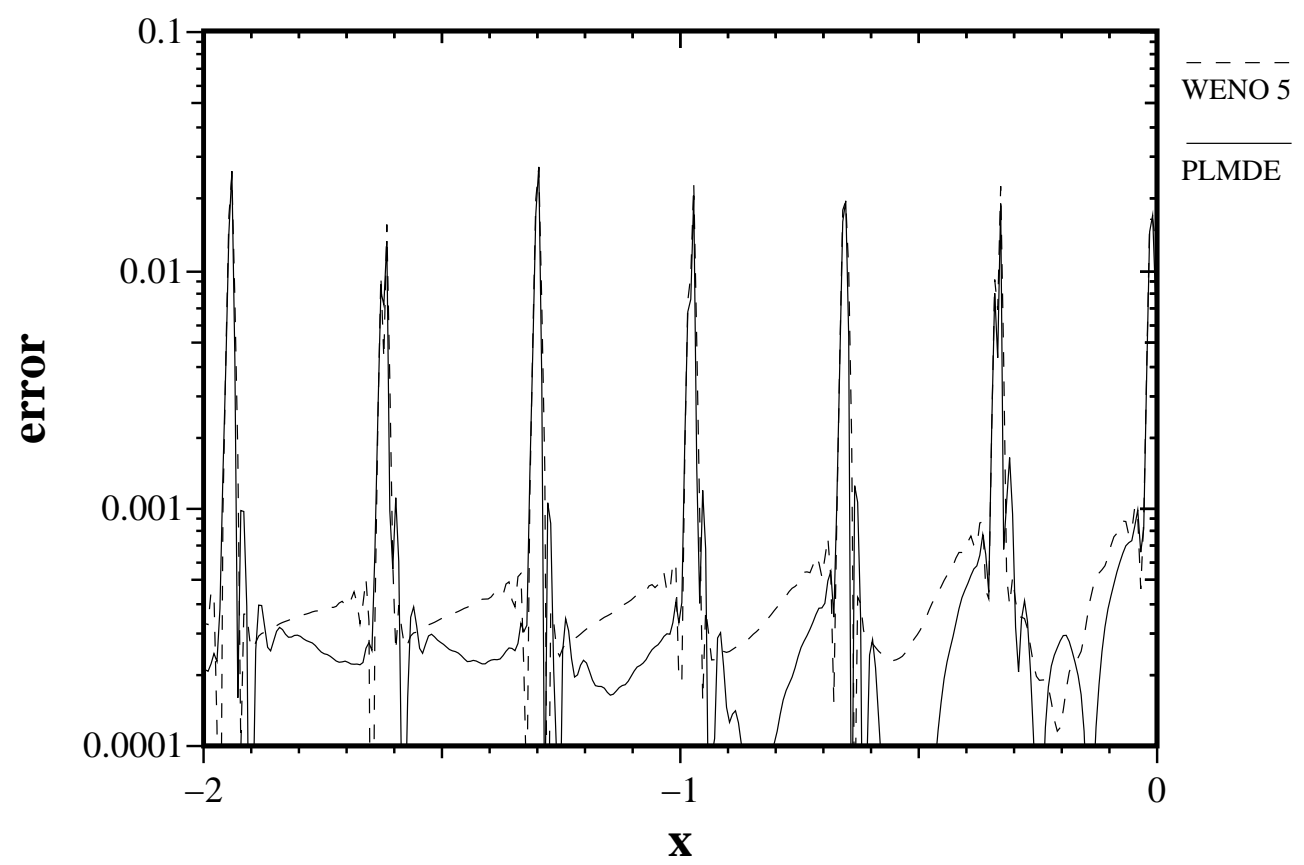

FIG. 8: A comparison of the errors in the far field (FF) region for the Shu and Osher test problem. PLMDE errors are shown as a solid line and WENO errors are shown with a dashed line.

The boundary conditions at $x=0$ and $x=1$ are set to solid reflecting walls. For a more detailed description of this problem and its evolution see [26]. Instead of following the evolution over time, we focus on the final time solution at $t=0.38$.

\section{Error Analysis}

For a solution as complex as this one, quantitative error measures are a necessity. The solution computed with PPMDE and $N=6400$ is used as the true solution for this problem. As with the previous shock-entropy problem, our first task is to provide the evidence necessary to provide confidence in this solution as an appropriate standard for comparison. In Table XVIII we display the $L_{1}$ and $L_{\infty}$ errors for this solution as well as the self-convergence rates. The $L_{1}$ errors are a factor of nearly 40 times lower than those estimated for PLMDE and WENO5 below. In the case of the $L_{\infty}$ errors this factor is nearly 30 . 


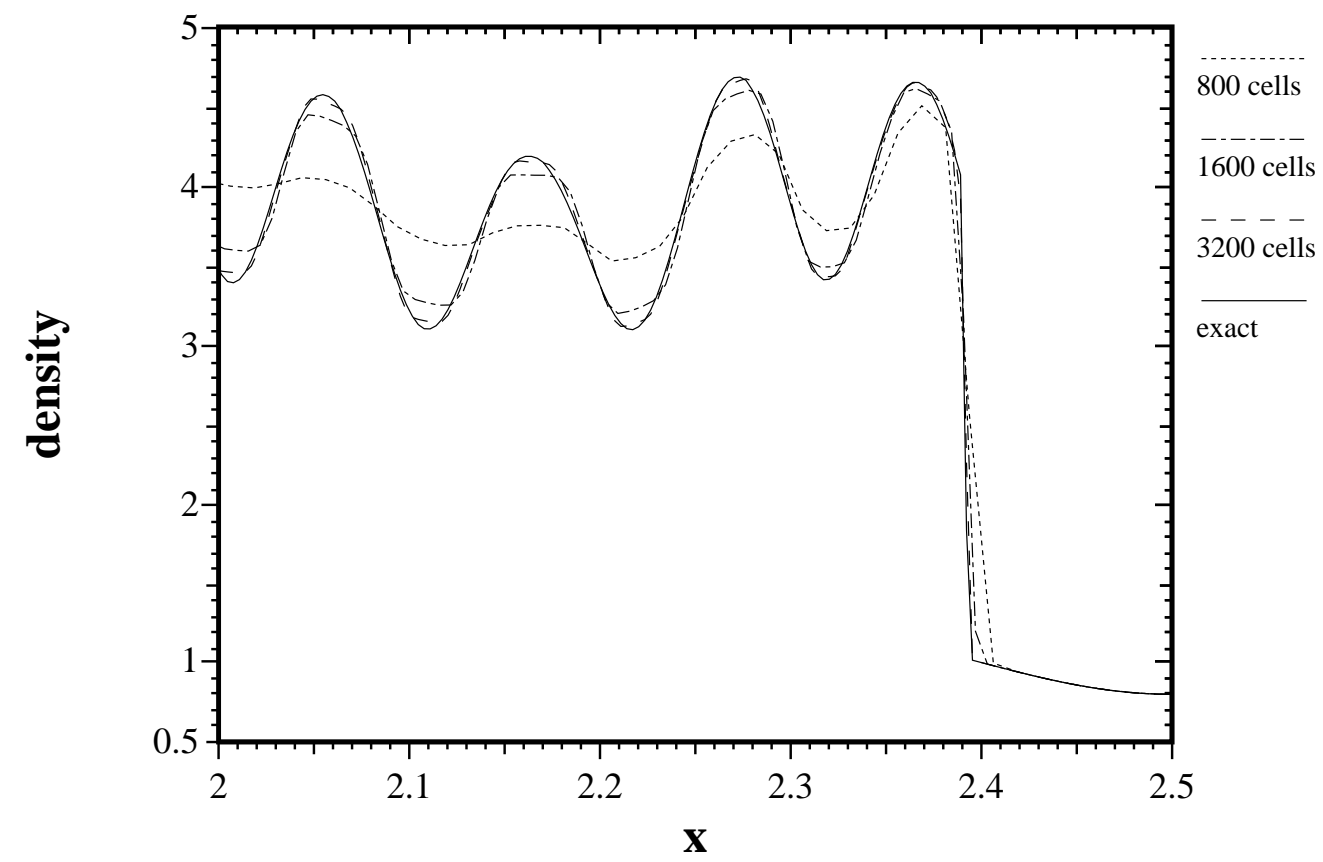

FIG. 9: The 800, 1600 and 3200 zone PLMDE solutions are shown in the near shock (NS) region for the Shu and Osher test problem.

TABLE XVIII: $L_{1}$ and $L_{\infty}$ Errors for the highly converged PPMDE solution to the interacting shock wave problem.

\begin{tabular}{|c|c|c|c|c|}
\hline $\mathrm{N}$ & $E_{L_{1}}$ & $L_{1}$ Rate & $E_{L_{\infty}}$ & $L_{\infty}$ Rate \\
\hline $1600: 3200$ & $1.93 \mathrm{e}-03$ & - & $1.92 \mathrm{e}-01$ & - \\
\hline $3200: 6400$ & $1.06 \mathrm{e}-03$ & 0.86 & $1.29 \mathrm{e}-01$ & 0.56 \\
\hline
\end{tabular}

In Table XIX, the $L_{1}$ errors are reported as well as the convergence rates. The overall magnitude of the errors for both methods are within $10-15 \%$ and the convergence rates are nearly first order. PLMDE is more accurate at all grid resolutions tested here. If instead we look at the $L_{\infty}$ norm errors shown in Table XX, we see that the overall error levels are high for both methods due to the presence of multiple flow discontinuities. The spatial distribution of error is displayed in Figure 17. The largest contribution to the error occurs at the contact discontinuity located near $x \approx 0.60$, that is embedded in a strongly rarefying flow. 


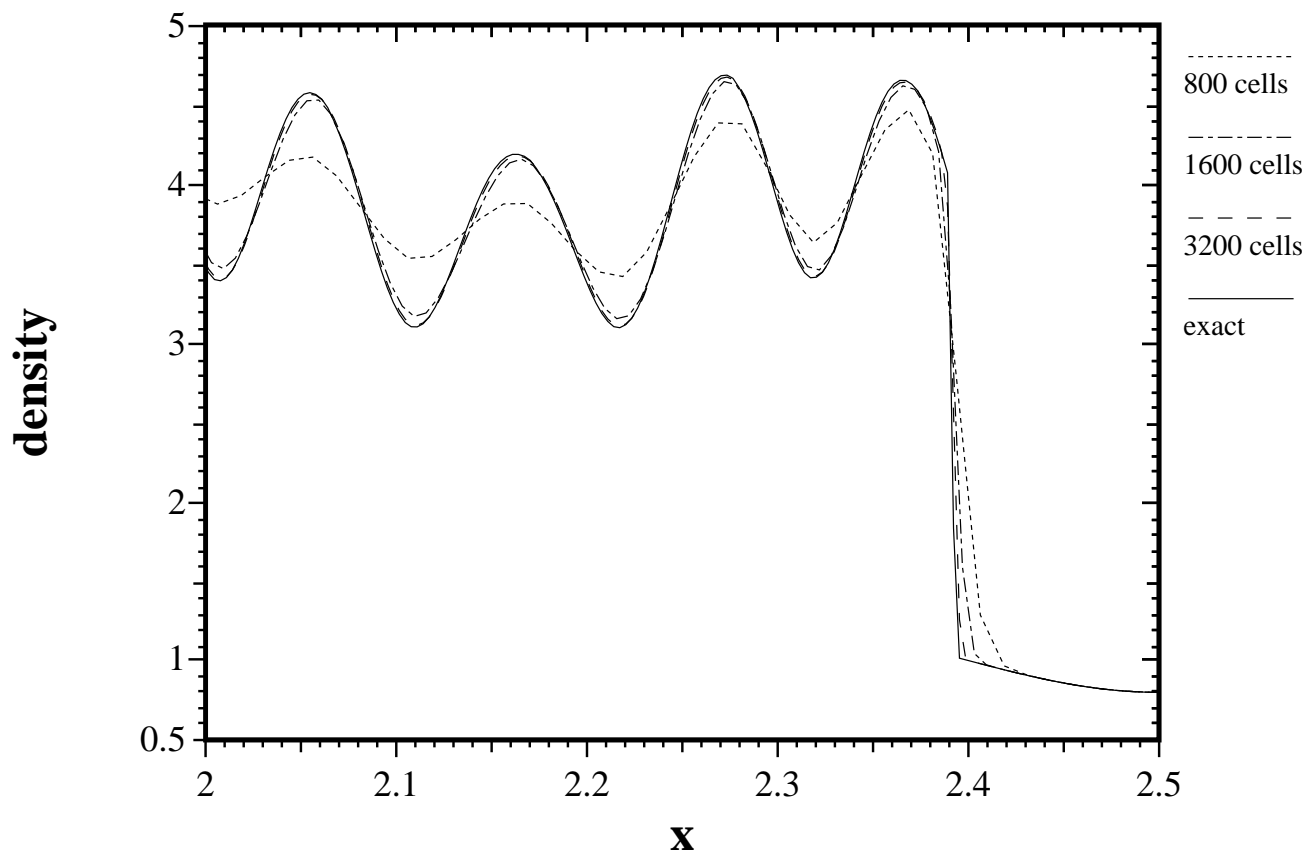

FIG. 10: The 800, 1600 and 3200 zone WENO solutions are shown in the near shock (NS) region for the Shu and Osher test problem.

TABLE XIX: $L_{1}$ error for PLMDE and WENO relative to the $N=6400$ PPMDE solution for the interacting shock wave problem.

\begin{tabular}{|c|c|c|c|c|}
\hline $\mathrm{N}$ & $E_{L_{1}}$ PLMDE & PLMDE Rate & $E_{L_{1}}$ WENO & WENO Rate \\
\hline 200 & $1.133 \mathrm{e}-01$ & - & $1.256 \mathrm{e}-01$ & - \\
\hline 400 & $6.298 \mathrm{e}-02$ & 0.85 & $7.071 \mathrm{e}-02$ & 0.83 \\
\hline 800 & $3.777 \mathrm{e}-02$ & 0.74 & $4.240 \mathrm{e}-02$ & 0.74 \\
\hline
\end{tabular}

\section{Break-even Analysis}

Reference to table XXI, it is clear that for the cost of the WENO solution, PLMDE can use a grid that is approximately 2.5 times as fine. That is, the $N=800$ PLMDE solution 


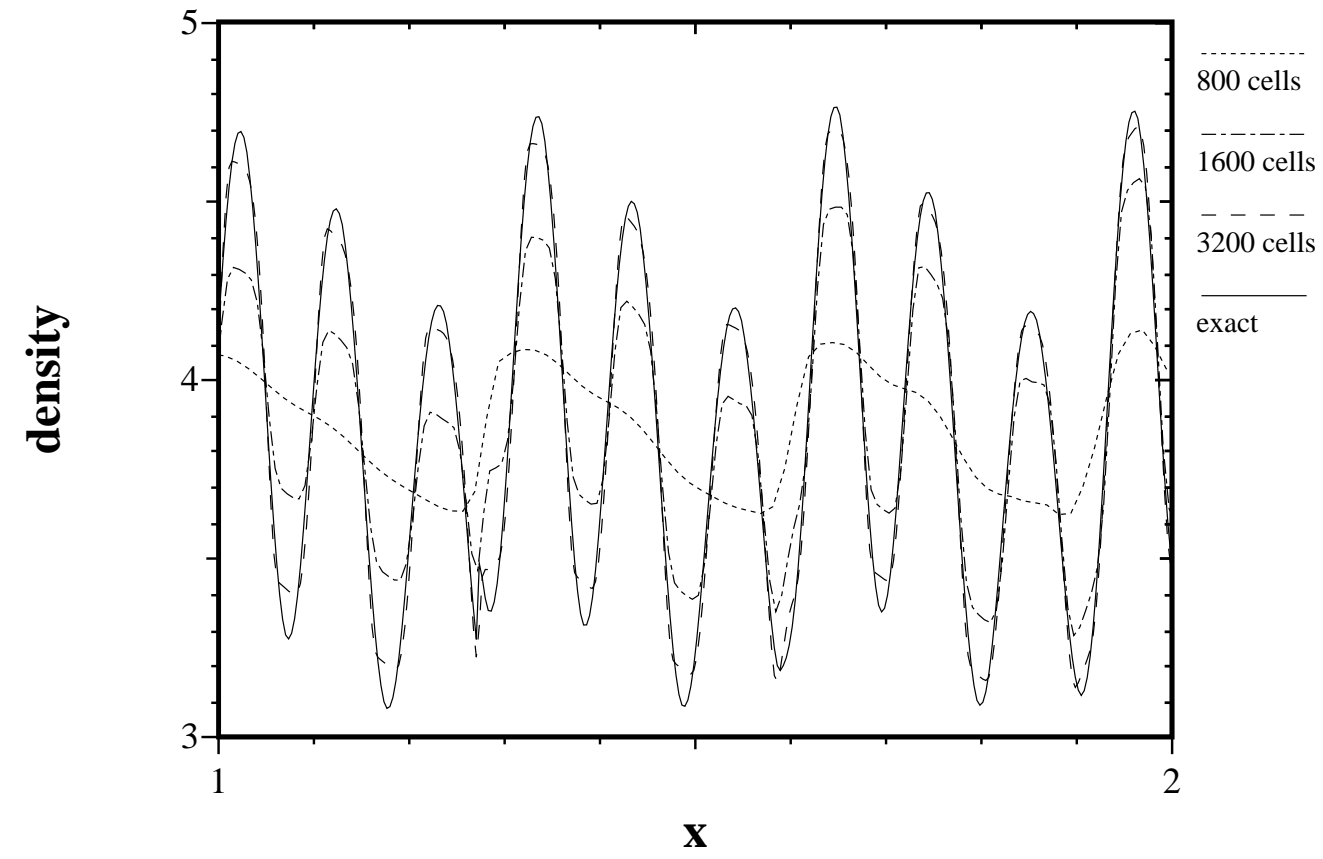

FIG. 11: The 800, 1600 and 3200 zone PLMDE solutions are shown in the entropy wave (EW) region for the Shu and Osher test problem.

TABLE XX: $L_{\infty}$ error for PLMDE and WENO for the interacting shock wave problem.

\begin{tabular}{|c|c|c|}
\hline $\mathrm{N}$ & $E_{L_{\infty}}$ PLMDE & $E_{L_{\infty}}$ WENO \\
\hline 200 & 3.126 & 3.298 \\
\hline 400 & 3.258 & 3.605 \\
\hline 800 & 3.274 & 3.740 \\
\hline
\end{tabular}

takes $16.91 \mathrm{~s}$ to compute the solution at $t=0.038$ while WENO using $N=300$ method takes $17.01 s$ to integrate to the final time. With the $L_{1}$ errors converging at a first order rate, this implies that 2.5 times the number of grid points for PLMDE will produce $L_{1}$ errors lower by a factor of two smaller than the WENO method. For just a factor of two increase in grid points, we see that this is true (cf. Table XIX). 


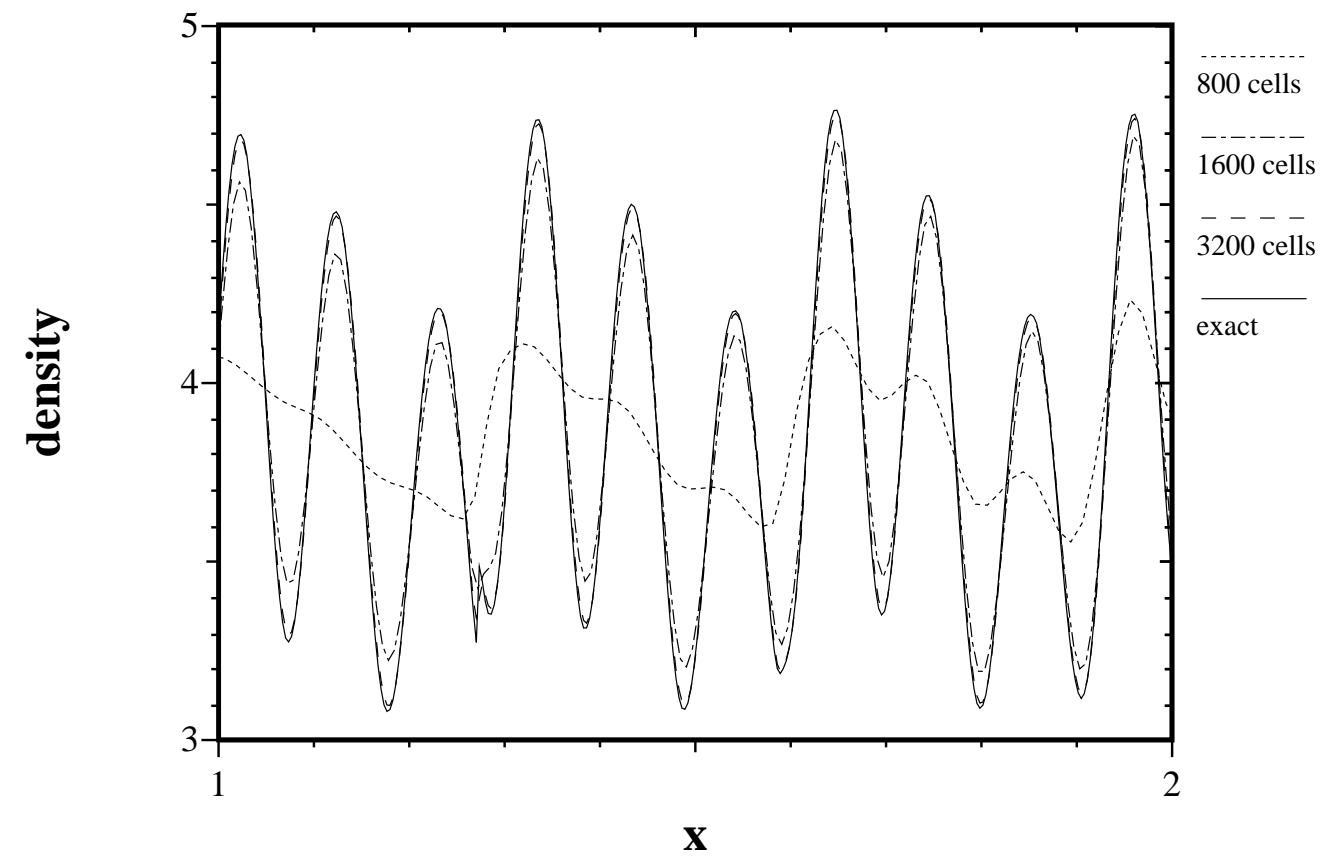

FIG. 12: The 800, 1600 and 3200 zone WENO solutions are shown in the entropy wave (EW) region for the Shu and Osher test problem.

\section{Density Plot Comparison}

In this section, we depict graphically the facts established for this test problem. We compare the density field computed by the WENO method at a particular resolution against the same resolution PLMDE solution and a PLMDE solution at twice the number of grid points. Figure 18 shows the comparison between with the WENO result at $N=200$ and a $N=200$ and $N=400$ Godunov solution. Comparing the results at the peaks $(x \approx 0.78$ and $x \approx 0.65)$ between the $N=200$ solutions shows that WENO is better at reproducing the peaks. The overall similarity between the $L_{1}$ and $L_{\infty}$, though, show that away from the peaks PLMDE must be generating smaller errors than WENO so that upon integration of the pointwise errors over the entire domain yields results that are similar. Even at this coarse resolution, we see that PLMDE with both $N=200$ and $N=400$ produces a less diffuse left-most contact region $(0.55<x<0.65)$ compared to WENO.

In figure 19, the $N=800$ WENO result is compared with the $N=800$ and $N=1600$ PLMDE result. Again when we compare the $N=800$ solutions, WENO is better at the 


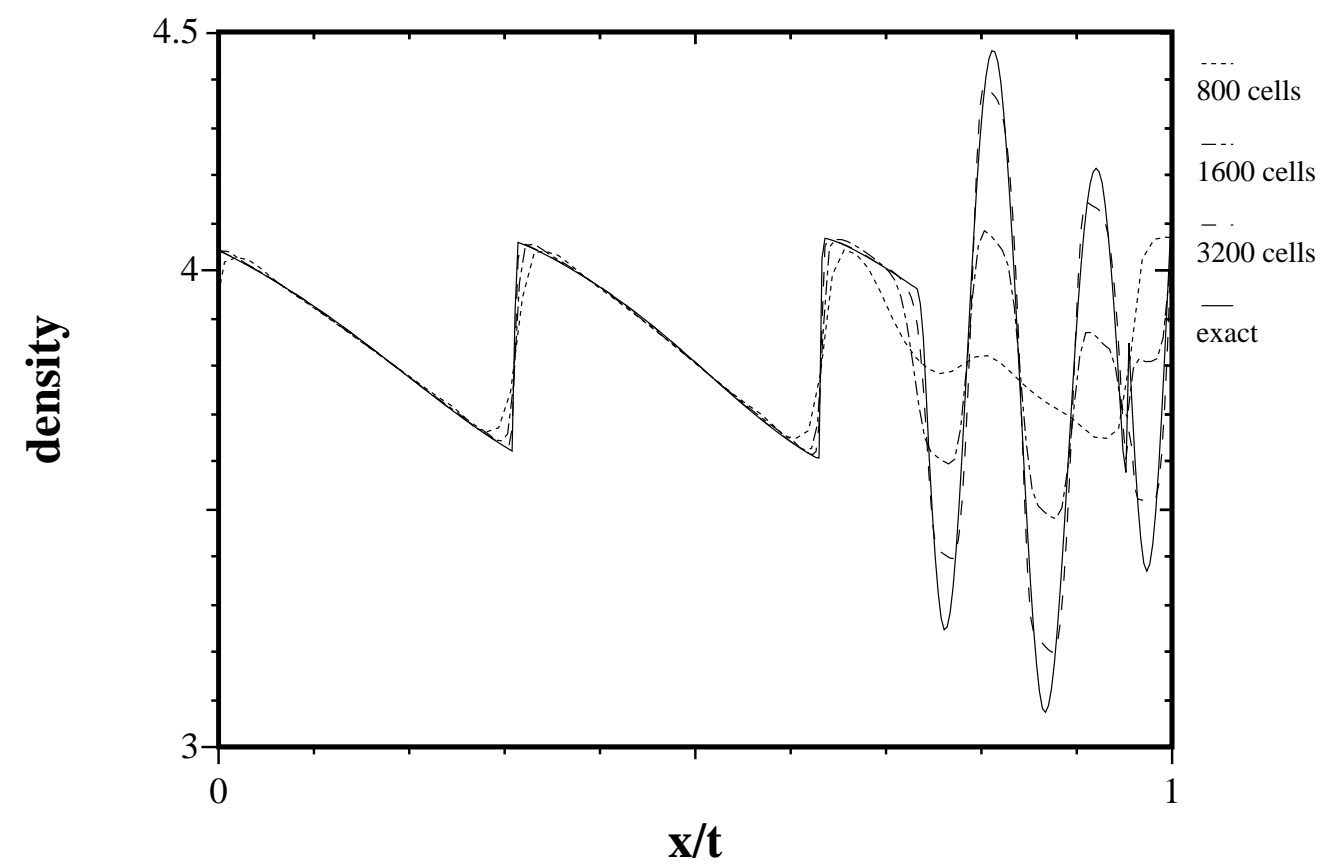

FIG. 13: The 800, 1600 and 3200 zone PLMDE solutions are shown in the transition region (TN) for the Shu and Osher test problem.

peaks, but this difference is much smaller than at coarser zoning. Still, the similarity of error norms at $N=800$ show that on average (after integration) PLMDE is producing an overall more accurate solution for this problem.

\section{Base Timings}

Timings in seconds for running the problem to $t=0.038$ on different grids are given in Table XXI. We use the same $C F L$ numbers as before. The following table contains the grid resolution used for both methods and the CPU time in seconds for PLMDE and for WENO in order to run the problem to $t=0.038$. We also list the ratio of the WENO time to PLMDE. Values greater than unity indicate that PLMDE is faster. These results are consistent with our earlier experience with these methods. 


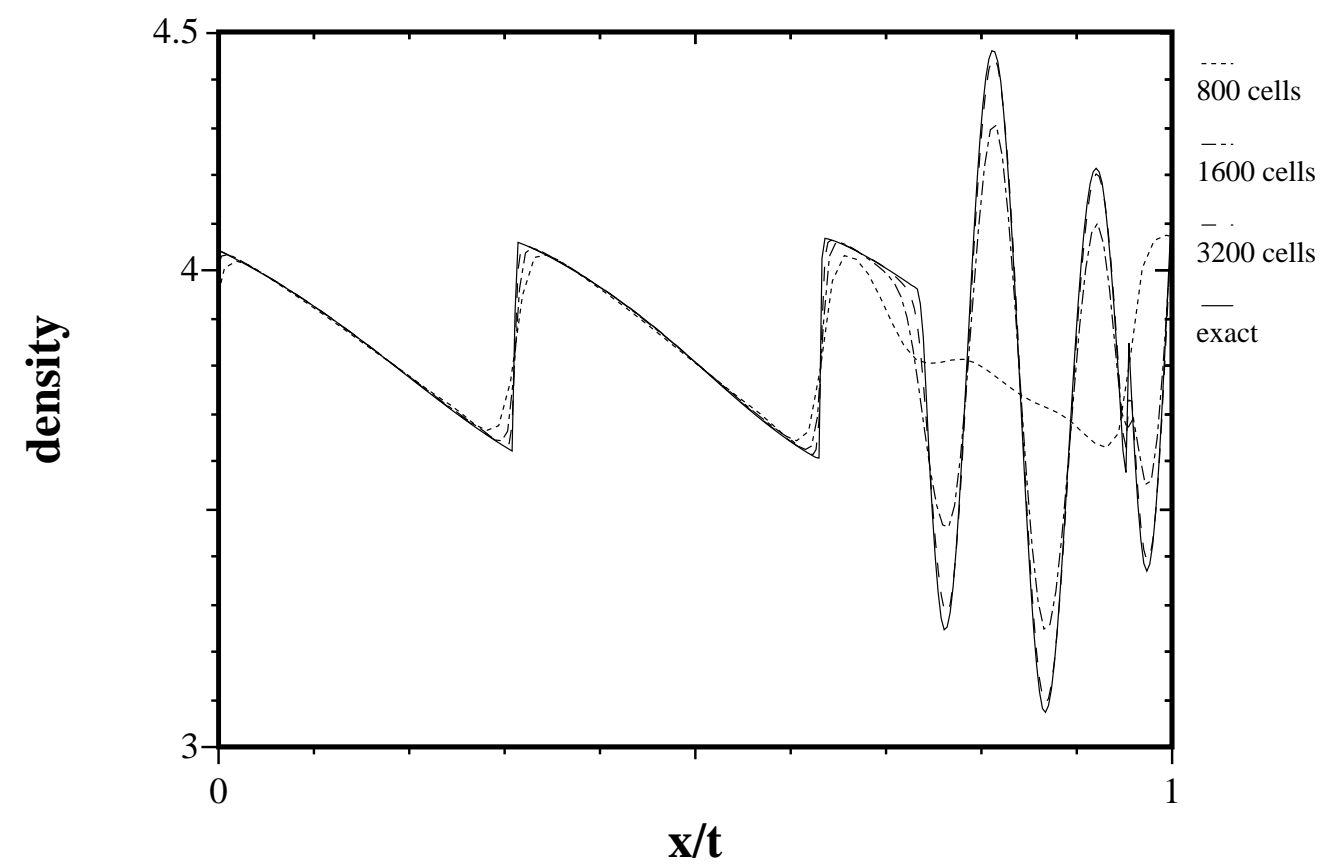

FIG. 14: The 800, 1600 and 3200 zone WENO solutions are shown in the transition region (TN) for the Shu and Osher test problem.

TABLE XXI: Timings for PLMDE and WENO on the interacting shock wave problem.

\begin{tabular}{|c|c|c|c|c|c|}
\hline $\mathrm{N}$ & PLMDE & time steps & WENO & time steps & Ratio \\
\hline 200 & 1.20 & 369 & 7.19 & 540 & 5.99 \\
\hline 400 & 4.29 & 723 & 27.80 & 1072 & 6.48 \\
\hline 800 & 16.91 & 1435 & 120.49 & 2141 & 7.13 \\
\hline
\end{tabular}

We note that it was found that a $C F L=1$ produced reasonable results for this test problem with WENO. In [13] the present problem was run at a $C F L=0.6$, so our timing results are set to be consistent with that practice.

\section{CONCLUSIONS}

This study has several important results.

- The most important of these is given the baseline performance of the WENO method 


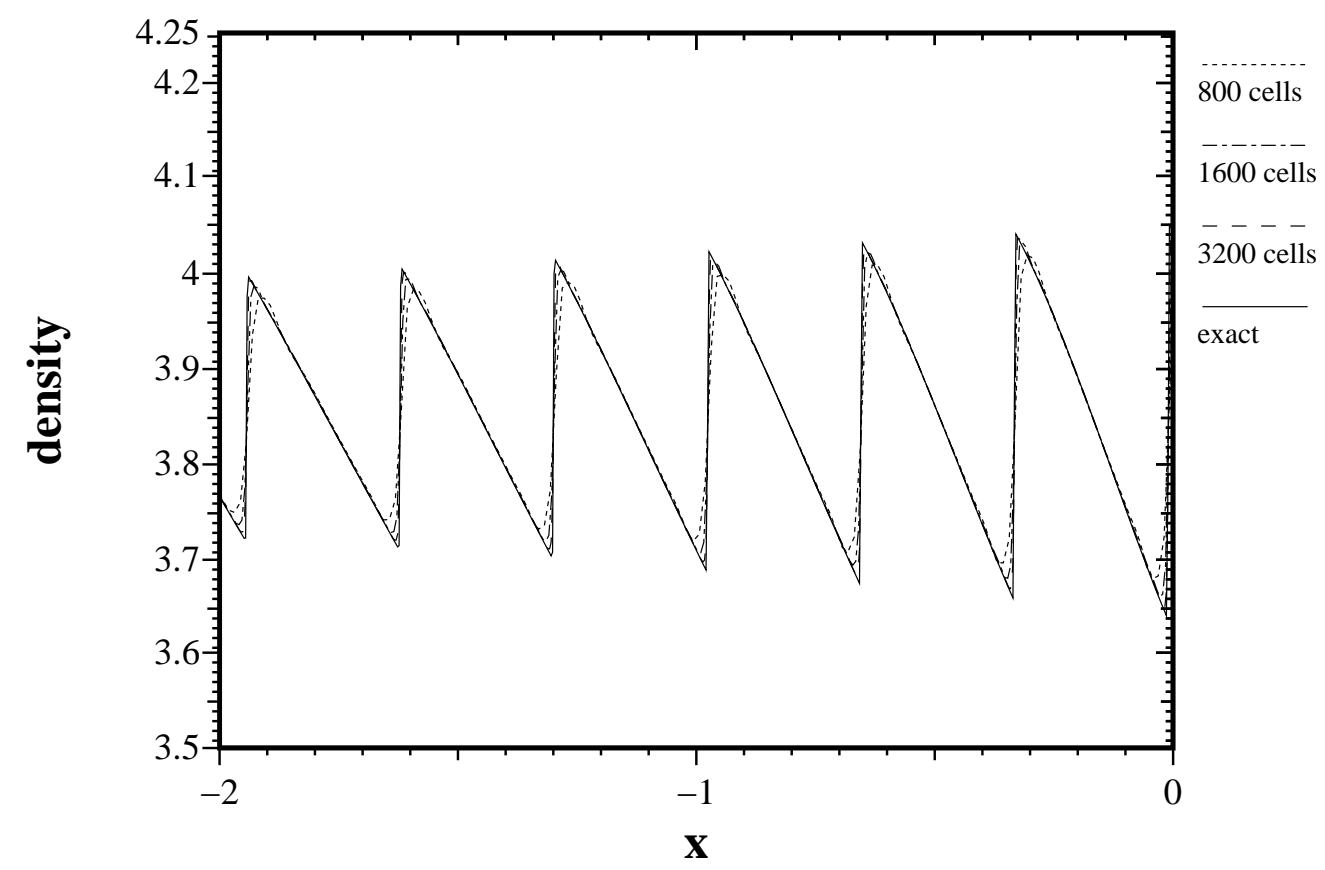

FIG. 15: The 800, 1600 and 3200 zone PLMDE solution in the far field (FF) region for the Shu and Osher test problem.

on linear problems where its fifth order convergence rate is manifest, for the nonlinear problems considered here, the accuracy advantage of the fifth-order method has essentially vanished and the convergence rate has been reduced to first order.

- At a fixed resolution the overall level of errors (accuracy per unit grid cell), for the norms used here, are typically similar for the problems considered. The one notable exception is the Shu-Osher problem in the EW regime, for which the WENO method produces $L_{1}$ errors lower by nearly a factor of two.

- Also, we have shown nominally first order convergence for the methods to a highly resolved third independent method.

- Our next important result concerns the difference in run times for the two methods. At a typical $C F L$ used for each method, PLMDE is six times faster than WENO5 for a given mesh and simulation end time. For the nonlinear problems considered, when the computational expense between PLMDE and WENO5 is equated, PLMDE 


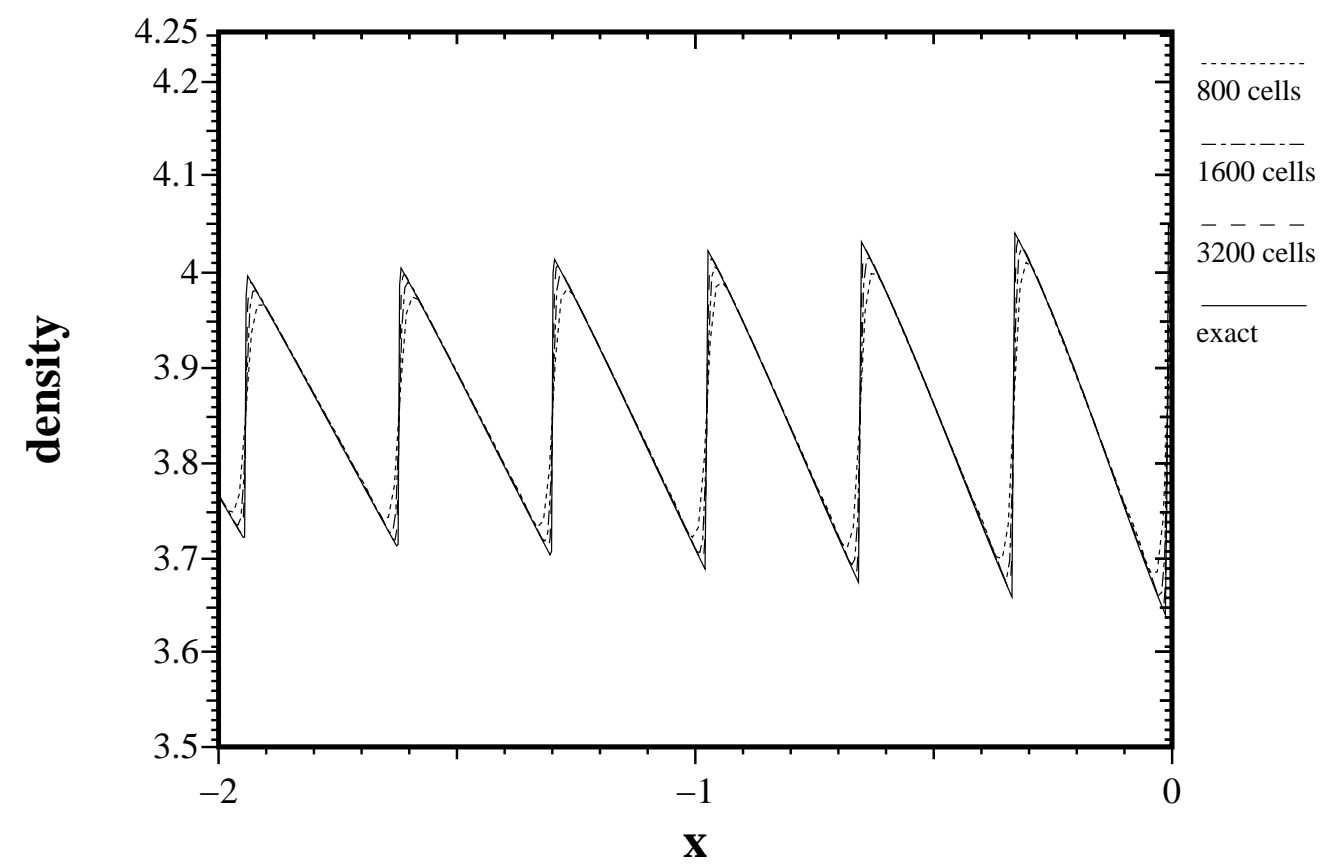

FIG. 16: The 800, 1600 and 3200 zone WENO solution in the far field (FF) region for the Shu and Osher test problem.

produces solutions with uniformly lower errors with respect to the norms considered (accuracy per unit CPU second).

This work has made clear progress in providing quantitative measures and comparisons between a formally high order accurate method and a formally second order one. It has demonstrated the importance of making detailed measurements of actual errors rather than simply relying upon qualitative comparisons between schemes. This allows both the relative strengths and weaknesses of these methods to be quantified unambiguously, so that sound conclusions can be drawn as to the efficacy of these methods on various classes of problems.

In light of these conclusions, an obvious question is raised: what has happened to the higher-order method's advantage observed in the linear regime when nonlinearity is introduced? While high-order convergence is not to be expected when intrinsic dissipation techniques are activated (e.g. when under-resolved gradients are present) as theoretical work predicts, it is generally hoped that the higher order method may offer lower overall errors. This was generally found to not be the case as the errors differed typically by only a factor 


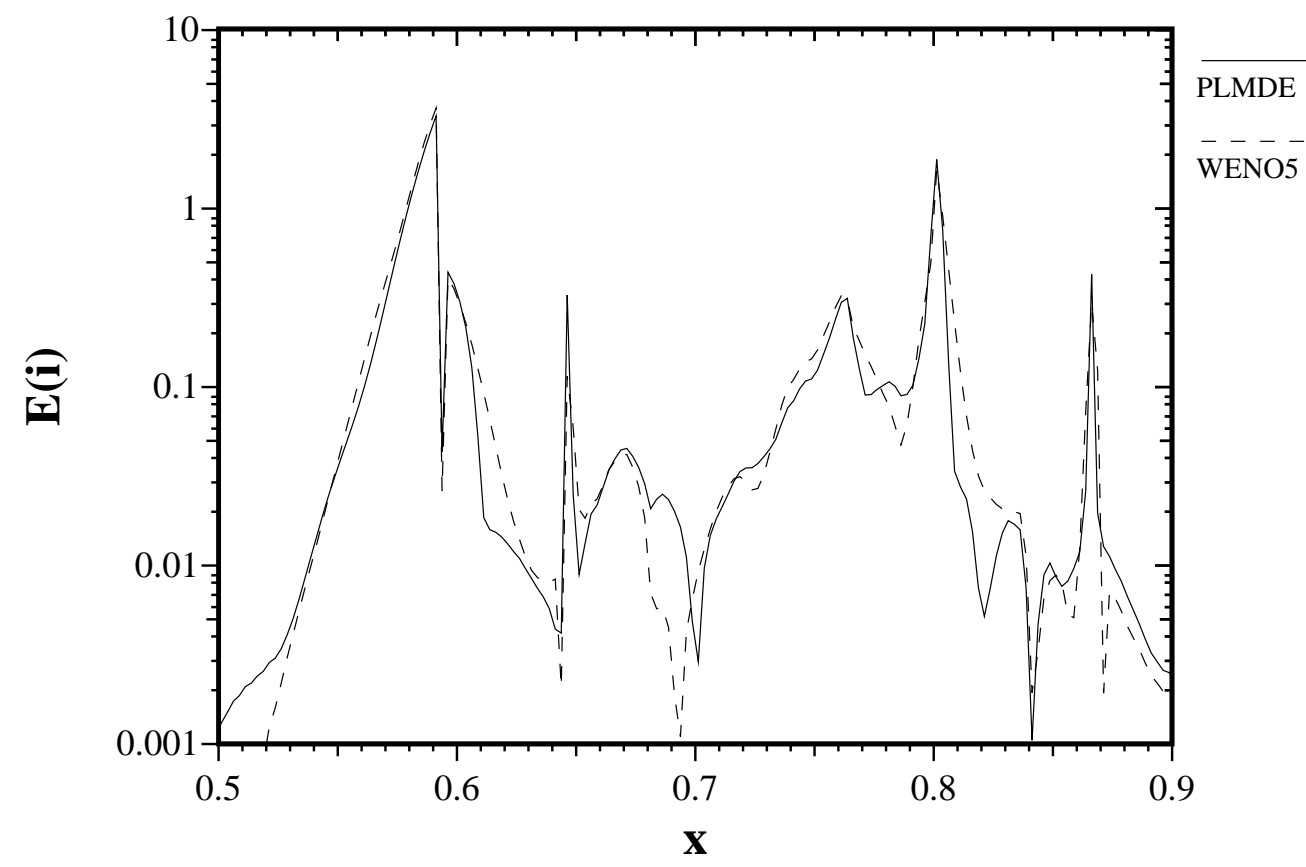

FIG. 17: A comparison of the spatial distribution of error for the interacting shock wave problem.

of two. The nonlinear problems considered here or other relevant ones should serve as tests to try to understand this issue more fully.

Two areas are ripe for further attention: (1) determining why the high order methods have lost the advantage so clear in the linear regime when nonlinearity comes into play, or equivalently, what parts of PLMDE make it particularly good for nonlinear problems; and (2) how does this picture change when multi-dimensional problems are considered? Some work in this area [8] indicates that the multi-dimensional results will be similar to those reported here; that is formally high order methods are comparable to the secondorder Godunov method for nonlinear problems in multiple spatial dimensions. Both of these areas are important and have potentially far reaching implications for the compressible flow simulation community.

\section{ACKNOWLEDGMENT}

This work was performed under the auspices of the U.S. Department of Energy by the University of California Lawrence Livermore and Los Alamos National Laboratories under 


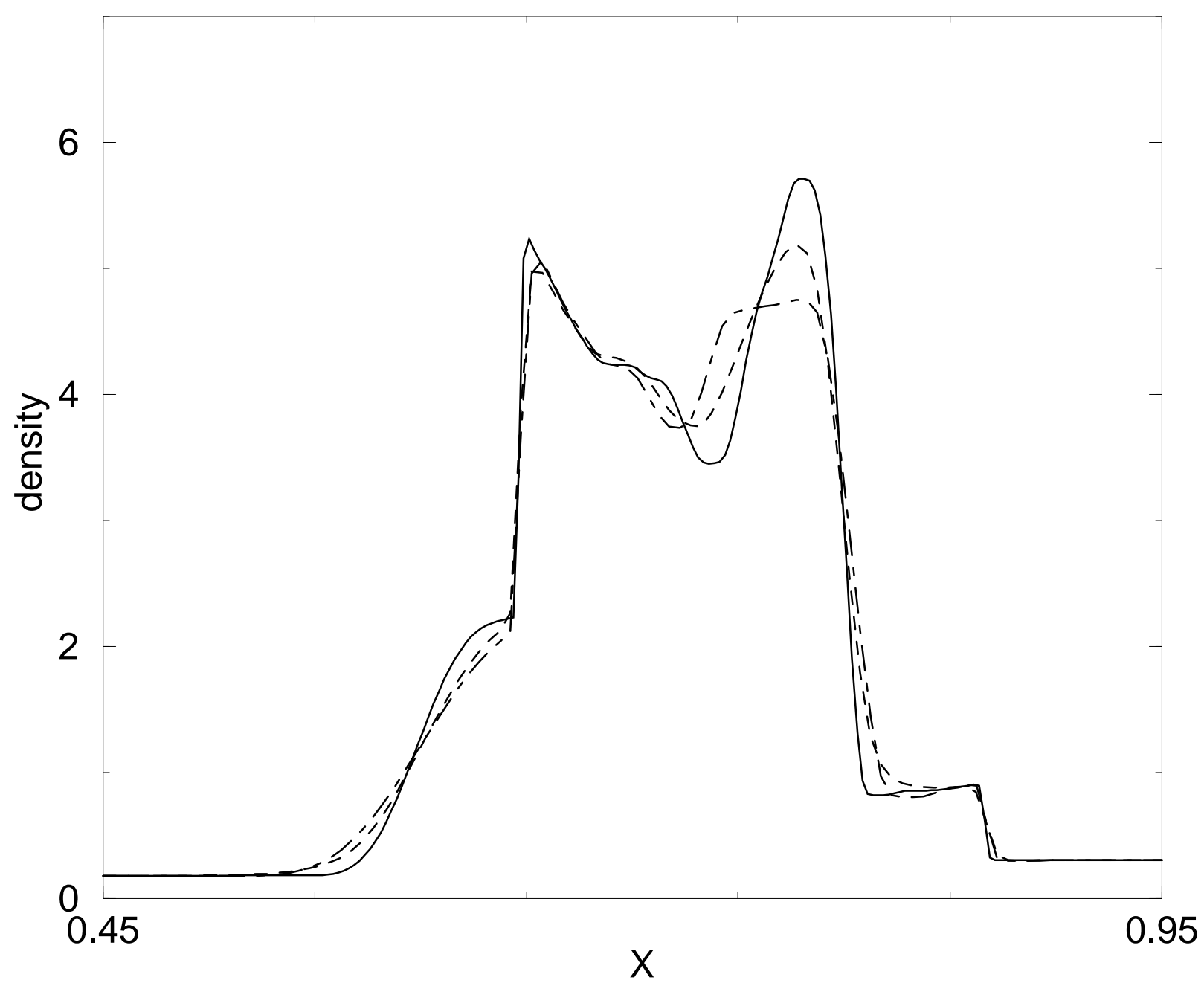

FIG. 18: The density at $t=0.038$ for the interacting shock wave test problem. The WENO result with $\mathrm{N}=200$ (dashed line) is shown with PLMDE results at $\mathrm{N}=200$ (dash-dot line) and at $\mathrm{N}=400$ (solid line).

contract No. W-7405-Eng-48.

[1] D. S. Balsara. Total variation diminishing scheme for adiabatic and isothermal magnetohydrodynamics. Astrophysical J. Supp. Ser., 116:133-153, 1998.

[2] J.B. Bell, P. Colella, and J. Trangenstein. Higher order godunov methods for general systems of hyperbolic conservation laws. J. Comput. Phys., 82:362-397, 1989.

[3] J. Casper and M. H. Carpenter. Computational considerations for the simulation of shockinduced sound. SIAM J. of Sci. Comp., 19(3):813-828, 1998. 


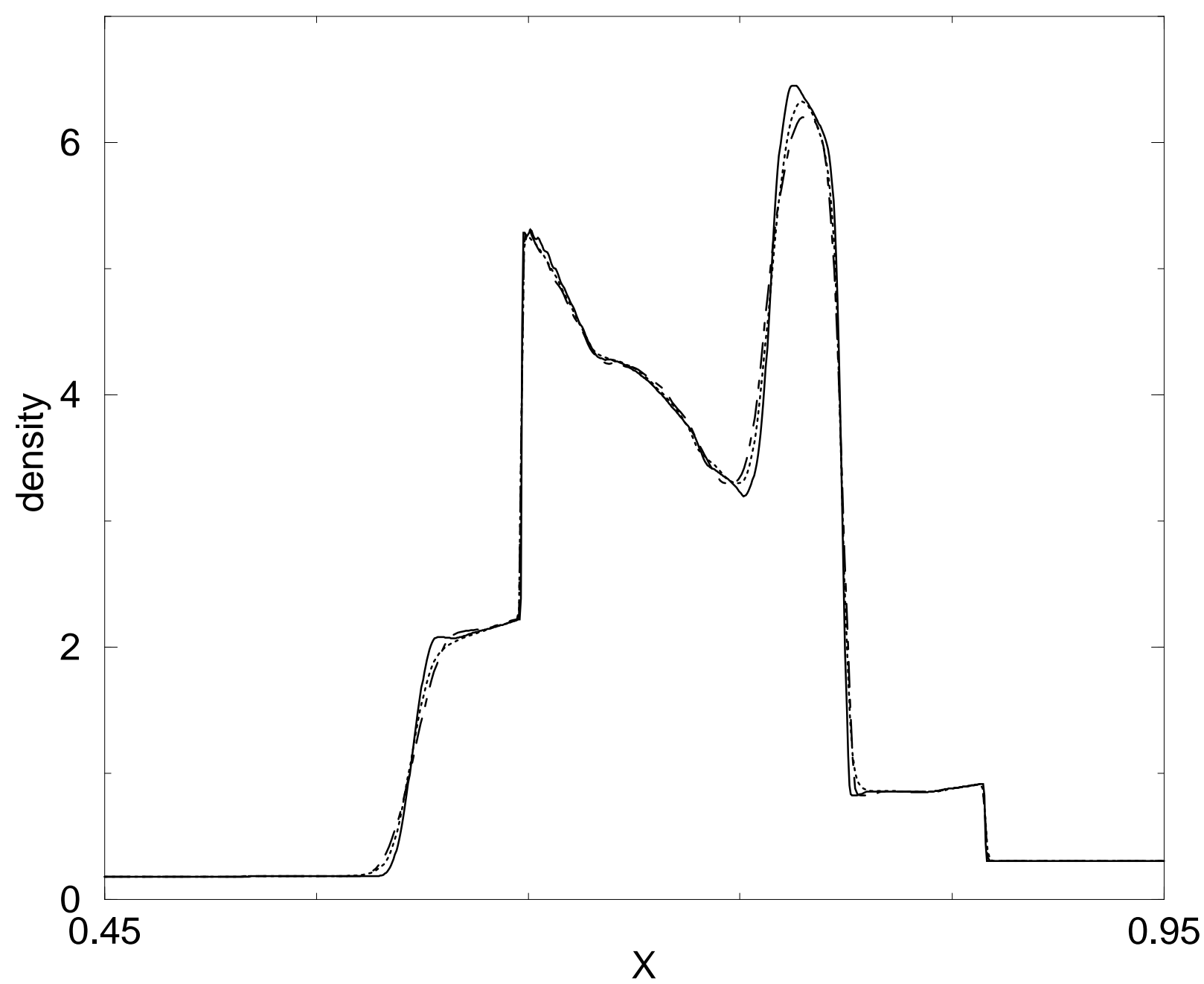

FIG. 19: The density at $t=0.038$ for the Interacting Shock Wave test problem. The WENO result with $\mathrm{N}=800$ (dashed line) is shown with the PLMDE results at $\mathrm{N}=800$ (dash-dot line) and at $\mathrm{N}=1600$ (solid line).

[4] P. Colella. A direct Eulerian MUSCL scheme for gas dynamics. SIAM J. Sci. Stat. Comput., 6:104-117, 1985.

[5] P. Colella. Multidimensional upwind methods for hyperbolic conservation laws. J. Comput. Phys., 87:171-200, 1990.

[6] P. Colella, H.M. Glaz, and R.E. Ferguson. Multifluid algorithms for Eulerian finite difference methods. unpublished, 2002.

[7] P. Colella and P. Woodward. The piecewise parabolic method (PPM) for gas-dynamical simulations. J. Comput. Phys., 54:174-201, 1984.

[8] A. W. Cook, W. H. Cabot, and J. A. Greenough. A comparison of high and low order schemes 
for shock-induced mixing. J. Comput. Phys., N/A:N/A, 2001. In review.

[9] R. Donat and S. Osher. Propagation of error in regions of smoothness for nonlinear approximations to hyperbolic equations. Comp. Meth. Appl. Mech. Engr., 80:59-64, 1990.

[10] S. Gottleib, C.-W. Shu, and E. Tadmor. Strongly stability preserving high-order time discretization methods. SIAM Review, 43:89-112, 2001.

[11] A. Harten, B. Engquist, S. Osher, and S. Chakravarthy. Uniformly high order accurate essentially non-oscillatory schemes, III. J. Comput. Phys., 71:231-303, 1987.

[12] H. T. Huynh. Accurate upwind methods for the Euler equations. SIAM J. Numer. Anal., 32:1565-1619, 1995.

[13] G-S. Jiang and C-W. Shu. Efficient implementation of weighted ENO schemes. J. Comput. Phys., 126:202-228, 1996.

[14] R. Liska and B. Wendroff. Comparison of several difference schemes on 1D and 2D test problems for the Euler equations. Technical Report LA-UR-01-6225, Los Alamos National Laboratory, 2001.

[15] R. Liska and B. Wendroff. Comparison of several difference schemes on 1D and 2D test problems for the Euler equations. SIAM Journal on Scientific Computing, In Press, 2003.

[16] X.-D. Liu, S. Osher, and T. Chan. Weighted essentially non-oscillatory schemes. J. Comput. Phys., 115:200-212, 1994.

[17] A. Majda and S. Osher. Propogation of error into regions of smoothness for accuracte difference approximations to hyperbolic equations. Comm. Pure Appl. Math., 30:671-705, 1977.

[18] G. H. Miller and E. G. Puckett. A higher-order Godunov method for multiple condensed phases. J. Comput. Phys., 128:134-164, 1996.

[19] J.S. Saltzman. An unsplit 3-D upwind method for hyperbolic conservation laws. J. Comput. Phys., 115:153-168, 1994.

[20] C.-W. Shu. Total-variation-diminishing time discretizations. SIAM J. Sci. Stat. Comp., 9:1073-1084, 1988.

[21] C.-W. Shu. Numerical experiments on the accuracy of ENO and modifed ENO schemes. Journal of Scientific Computing, 5:127-151, 1990.

[22] C.-W. Shu and S. J. Osher. Efficient implementation of essentially non-oscillatory shock capturing schemes ii. J. Comput. Phys., 83:32-78, 1989.

[23] G. Sod. A survey of several finite difference methods for systems of nonlinear hyperbolic 
conservation laws. J. Comput. Phys., 27:1-31, 1978.

[24] A. Suresh and H. T. Huynh. Accurate monotonicity-preserving schemes with Runge-Kutta time stepping. J. Comput. Phys., 136:83-99, 1997.

[25] B. van Leer. Towards the ultimate conservative difference scheme. V. A second-order sequel to Godunov's method. J. Comput. Phys., 32:101-136, 1979.

[26] P. Woodward and P. Colella. The numerical simulation of two-dimensional fluid flus with strong shocks. J. Comput. Phys., 54:115-173, 1984.

[27] S. T. Zalesak. A preliminary comparison of modern shock-capturing schemes: Linear advection. In R. Vichnevetsky and R. S. Stepleman, editors, Advances in Computer Methods for Partial Differential Equations, volume 6, pages 15-22. IMACS, 1987.

[28] PLMDE is an acronym for Piecewise Linear MUSCL Direct Eulerian. This terminology is inherited from the distinction between methods based upon a Lagrangian-Remap (LR) step. For example, van Leer's extension of Godunov's original method would be termed as PLMLR. This distinction can be most clearly seen in Woodward and Colella's review paper [7, 26] with the piecewise parabolic MUSCL (PPMLR or PPMDE). 